ESAIM: M2AN 47 (2013) 83-108

DOI: $10.1051 / \mathrm{m} 2 \mathrm{an} / 2012020$
ESAIM: Mathematical Modelling and Numerical Analysis

www.esaim-m2an.org

\title{
THE EXTENDED ADJOINT METHOD
}

\author{
Stanislas Larnier ${ }^{1}$ And Mohamed Masmoudi ${ }^{1}$
}

\begin{abstract}
Searching for the optimal partitioning of a domain leads to the use of the adjoint method in topological asymptotic expansions to know the influence of a domain perturbation on a cost function. Our approach works by restricting to local subproblems containing the perturbation and outperforms the adjoint method by providing approximations of higher order. It is a universal tool, easily adapted to different kinds of real problems and does not need the fundamental solution of the problem; furthermore our approach allows to consider finite perturbations and not infinitesimal ones. This paper provides theoretical justifications in the linear case and presents some applications with topological perturbations, continuous perturbations and mesh perturbations. This proposed approach can also be used to update the solution of singularly perturbed problems.
\end{abstract}

Mathematics Subject Classification. 49Q10, 49Q12, 74P10, 74P15.

Received November 18, 2011. Revised April 18, 2012.

Published online July 31, 2012.

\section{INTRODUCTION}

The optimal partitioning of a domain $\Omega$ has important real-life applications like shape optimal design, detection of inclusions, image classification and segmentation. Topological derivative methods have been used to solve this kind of problems $[10,11,23,25,26,30,31,35,36]$. These approaches have some drawbacks:

- the asymptotic topological expansion is not easy to obtain for complex problems;

- it needs to be adapted for many particular cases like the creation of a hole on the boundary of an existing one or on the original boundary of the domain;

- we don't know how to calculate the variation of a cost function when a hole is to be filled;

- in real applications of topology optimization, a finite perturbation is performed and not an infinitesimal one such as an element deletion in a mesh.

Certain issues arise here, as for example the question on how large the topological change should be. In the present paper, we propose an extension of the adjoint method to overcome these problems.

The problem of optimal partitioning of a domain $\Omega$ is equivalent to the problem of looking for an optimal function $c$ which takes a finite number of values $0,1, \ldots, m-1$. If $m=2, c$ is the characteristic function of an unknown optimal subdomain of $\Omega$. For the sake of simplicity we will limit the study to the case where $m=2$.

\footnotetext{
Keywords and phrases. Adjoint method, topology optimization, calculus of variations.

1 Université Paul Sabatier, Institut de Mathématiques de Toulouse, 118 route de Narbonne, 31062 Toulouse, France.

stanislas.larnier@math.univ-toulouse.fr; mohamed.masmoudi@math.univ-toulouse.fr
} 
Even if we are dealing with a $0-1$ optimization problem, it is possible to use variational methods. For a cost function

$$
\begin{aligned}
j: L^{p}(\Omega) & \rightarrow \mathbb{R}, 1 \leq p<\infty \\
c & \mapsto j(c),
\end{aligned}
$$

the topological asymptotic expansion consists in calculating the variation of $j$ when $c$ switches from 1 to 0 or from 0 to 1 in a small area. Generally, $j$ depends on $c$ via the resolution of a set of partial differential equations. Explicit regularization terms related to the volume, or the measure of the boundary, as well as the mean curvature, could be added to $j$ and a more regular space than $L^{p}$ could be considered.

It is possible to use differential calculus tools [27] for estimating the variation of $j$ when $c$ switches from 1 to 0 or from 0 to 1 in a small region $\omega_{\varepsilon}=x_{0}+\varepsilon \omega$, where $x_{0}$ is a point of $\Omega, \varepsilon$ is a small scalar, and $\omega$ is a given domain. The perturbation $\delta c= \pm \chi_{\omega_{\varepsilon}}$ obtained when $c$ switches from 1 to 0 or from 0 to 1 in a small region $\omega_{\varepsilon}$, is small in $L^{p}(\Omega)$ even if not small in magnitude. We will refer to $\delta c$ as a singular perturbation. If $j$ is differentiable, we have

$$
\left|j(c+\delta c)-j(c)-\int_{\Omega} g_{1} \delta c\right|=\mathcal{O}\left(\|\delta c\|_{L^{p}}^{2}\right),
$$

where $g_{1} \in L^{q}(\Omega), \frac{1}{p}+\frac{1}{q}=1$ is the gradient of $j$. Moreover, if $g_{1}$ is regular, we obtain the following expansion:

$$
|j(c+\delta c)-j(c)-| \omega_{\varepsilon}\left|g_{1}\left(x_{0}\right)\right|=\mathcal{O}\left(\left|\omega_{\varepsilon}\right|^{\frac{2}{p}}\right)+\circ\left(\left|\omega_{\varepsilon}\right|\right)
$$

There are two cases:

- when $p<2$, the rest in equation (1.1) is of higher order and the topological gradient is equal to $g_{1}$, the gradient of $j$. We refer to [27] for more details;

- when $p \geq 2$, the quantity $g_{1}$ is the first term of the topological gradient, the "rest" $\mathcal{O}\left(\left|\omega_{\varepsilon}\right|^{\frac{2}{p}}\right)$ could contribute to the topological gradient by a second (or hidden) term $g_{2}$.

The goal of this paper is to provide a simple, efficient and general way to calculate the second term $g_{2}$. In the case $p \geq 2$, the asymptotic expansion (1.1) is technically correct, but it may lead to wrong conclusions.

The adjoint method is the classical way to calculate $g_{1}$, the gradient of $j$, at each point of the domain $\Omega$ and at a lower computing cost. In fact, at least for the case $p<2$, there is no need to calculate the variation of the state with respect to $c$, because its contribution is of higher order. And when $p \geq 2$, the variation of the solution gives the second term $g_{2}$ of the topological gradient. In simple cases, this second term $g_{2}$ is quite easy to estimate using the explicit knowledge of the fundamental solution of the problem and there is no need to calculate the unknown variation of the state with respect to the considered perturbation $[1,10,11,23,25,26,30,31,35,36]$. In this case, the formal application of the chain rule gives only the first term. In some cases, such as the insertion of infinitesimal cracks [11], since $\left|\omega_{\varepsilon}\right|=0$, the first term is equal to zero and the variation of the cost function is equal to the hidden contribution. Currently, some authors such as Bonnet are interested by asymptotic topological expansions of higher-order [17,18].

Like we said before, these methods have many limitations. The fundamental solution is not known explicitly for more realistic models. The asymptotic expansion needs to be adapted for too many particular cases like the creation of a hole on the boundary of an existing one or on the original boundary of the domain. The number of particular cases increases if the type of boundary conditions on the hole is considered. In [34], the case of the Laplace equation with the creation of a hole on a polygonal boundary has been studied. In topology optimization, we need to know how to fill an existing small hole, a problem studied by Guillaume and Hassine [24].

In most contributions, the elementary solution is used to calculate the local variation of the state around the singular perturbation. The key idea of this paper is to take into account this variation by solving a local perturbed problem defined in a small fixed domain $D$ containing the singular perturbation. The state of the initial uniform problem is imposed as a Dirichlet condition on the boundary of $D$. The size of the domain $D$ varies with the studied problem. 
From the application point of view, this method is non invasive and can be easily adapted to any solver. It could be used with parallel computing algorithms when multiple evaluations are needed.

From the theoretical point of view, this method could be seen as an improvement of the domain truncation method, where the variation of the Dirichlet to Neumann operator is needed $[10,23,30]$. Here we just need to study the variation of a local solution. Thus, our method could be a tool in theoretical investigations. This paper presents theoretical justifications and examples in the linear case. In [29], the method has been used in a nonlinear case with mesh perturbations in fluids dynamics.

This extension of the adjoint method is called the numerical vault for two reasons. The imposed Dirichlet condition on the boundary of $D$ makes our solution very stable and recalls the vault in architecture which is supported by its surrounding walls. Moreover, similarly to the vault, our method is very simple and has great potential for applications.

Section 2 recalls the adjoint technique and presents the basic concept of the vault method. In Section 3, we consider the linear case and present a theoretical justification for the numerical vault as an extension to the adjoint method. Some simple examples are also considered to illustrate the theoretical study. In Section 4, we show that the numerical vault is not limited to the estimation of the variation of a cost function, but can be applied to update the solution of a singularly perturbed problem. In Section 5, we present some applications of the numerical vault to topological perturbations and we show that the hidden term $g_{2}$ is not small in comparison to $g_{1}$. Moreover, in the case of a continuous perturbation, the numerical vault allows us to obtain a higher order behavior. We applied our method to continuous material properties and mesh perturbations. An application to elastography is presented in Sections 6 and 7, the numerical vault is applied to update the solution in the case of an image restoration problem.

\section{ThE ADJOINT METHOD}

\subsection{The adjoint technique}

We first recall the adjoint method in a formal way. Consider the following steady state equation

$$
F(c, u)=0 \text { in } \Omega,
$$

where $c$ is a distributed parameter in a domain $\Omega$. The aim is to minimize a cost function $j(c):=J\left(u_{c}\right)$ where $u_{c}$ is the solution of equation (2.1) for a given $c$.

Let us suppose that every term is differentiable. We are considering a perturbation $\delta c$ of the parameter $c$.

Since $c$ is a distributed parameter, its discretization leads to a huge vector. A fast gradient computation is therefore of high importance.

Equation (2.1) can be seen as a constraint, and as a consequence, the Lagrangian is considered:

$$
\mathcal{L}(c, u, p)=J(u)+(F(c, u), p),
$$

where $p$ is a Lagrange multiplier and $(\cdot, \cdot)$ denotes the scalar product in a well-chosen Hilbert space.

To compute the derivative of $j$, one can remark that $j(c)=\mathcal{L}\left(c, u_{c}, p\right)$ for all $c$, if $u_{c}$ is the solution of equation (2.1). The derivative of $j$ is then equal to the derivative of $\mathcal{L}$ with respect to $c$ :

$$
d_{c} j(c) \delta c=\partial_{c} \mathcal{L}\left(c, u_{c}, p\right) \delta c+\partial_{u} \mathcal{L}\left(c, u_{c}, p\right) \partial_{c} u \delta c .
$$

All these terms can be calculated easily, except $\partial_{c} u \delta c$, the solution of the linearized problem:

$$
\partial_{u} F\left(c, u_{c}\right)\left(\partial_{c} u \delta c\right)=-\partial_{c} F\left(c, u_{c}\right) \delta c .
$$

To avoid the resolution of this equation for each $\delta c$, the term $\partial_{u} \mathcal{L}\left(c, u_{c}, p\right)$ is cancelled by solving the following adjoint equation in $p$. Let $p_{c}$ be the solution of the adjoint equation:

$$
\partial_{u} F\left(c, u_{c}\right)^{T} p_{c}=-\partial_{u} J^{T} .
$$


So the derivative of $j$ is explicitly given by

$$
d_{c} j(c) \delta c=\partial_{c} \mathcal{L}\left(c, u_{c}, p_{c}\right) .
$$

This method works only when the problem is differentiable and $\delta c$ is an admissible perturbation. Several counter-examples, where one of these conditions is not satisfied, are given in [27].

\subsection{Generalized adjoint technique}

Note that if the Lagrangians $\mathcal{L}(c+\delta c, \ldots, \ldots)$ and $\mathcal{L}(c, \ldots, \ldots)$ are defined on the same space, we have

$$
j(c+\delta c)-j(c)=\mathcal{L}\left(c+\delta c, u_{c+\delta c}, p_{c}\right)-\mathcal{L}\left(c, u_{c}, p_{c}\right),
$$

it can be split in two terms:

$$
j(c+\delta c)-j(c)=\left(\mathcal{L}\left(c+\delta c, u_{c+\delta c}, p_{c}\right)-\mathcal{L}\left(c+\delta c, u_{c}, p_{c}\right)\right)+\left(\mathcal{L}\left(c+\delta c, u_{c}, p_{c}\right)-\mathcal{L}\left(c, u_{c}, p_{c}\right)\right) .
$$

In the case of a regular perturbation $\delta c$, the second term gives the main variation and the first term is of higher order. In the case of a singular perturbation, the first term is of the same order as the second one and cannot be ignored. Then the variation of $u_{c}$ has to be estimated.

In the topology optimization papers $[10,11,13,23,25,26,30]$, the local variation of $u_{c}$ can be expressed thanks to the elementary solution of the problem to solve. This solution is singular at $x_{0}$ and gives the local variation of $u_{c}$ around $x_{0}$. In general, it is not always simple to get an explicit expression of the elementary solution. This is particularly true for problems with discontinuous coefficients, if the discontinuity is located at $x_{0}$.

Due to this difficulty, a numerical estimation of this variation is presented. The basic idea of the numerical vault is to update the solution $u_{c}$ by solving a local problem defined in a small domain around $x_{0}$.

If the Lagrangians $\mathcal{L}(c+\delta c, \ldots, \ldots)$ and $\mathcal{L}(c, \ldots, \ldots)$ are not defined on the same space, we have

$$
j(c+\delta c)-j(c)=\mathcal{L}\left(c+\delta c, u_{c+\delta c}, p_{c+\delta c}\right)-\mathcal{L}\left(c, u_{c}, p_{c}\right) .
$$

In this case, the direct solution $u_{c}$ and also the adjoint $p_{c}$ are updated with the numerical vault.

\section{Estimation of THE VARIATION OF A COST FUNCTION With THE NUMERICAL VAULT}

For the sake of simplicity, only the linear case is studied. Consider the variational problem depending on a parameter $\varepsilon$

$$
a^{\varepsilon}(u, v)=\ell^{\varepsilon}(v) \quad \forall v \in \mathcal{V}^{\varepsilon},
$$

where $\mathcal{V}^{\varepsilon}$ is a Hilbert space, $a^{\varepsilon}$ is a bilinear, continuous and coercive form and $\ell^{\varepsilon}$ is a linear and continuous form. Typically, $\mathcal{V}^{\varepsilon}$ is such that $\mathrm{H}_{0}^{1} \subset \mathcal{V}^{\varepsilon} \subset \mathrm{H}^{1}$.

In this study, a singular perturbation means a finite variation located in an infinitesimal area: the size of the perturbation goes to zero when $\varepsilon$ goes to zero. Depending on the problem, $\varepsilon$ can be the thickness of a coating layer around a variety of dimension $\leq n-1$ in the space of dimension $n$. In the case of a hole, $\varepsilon$ can be the diameter of a tube around a curve in the space of dimension 3. And we do not forget the classical case, where $\varepsilon$ is the diameter of a hole created around a point $x_{0}$.

Let $u^{\varepsilon}$ be the solution of the problem (3.1) and $u^{0}$ be the solution of the initial problem without any perturbation at $\varepsilon=0$.

The objective is to calculate the variation, with respect to $\varepsilon$, of

$$
j(\varepsilon):=J^{\varepsilon}\left(u^{\varepsilon}\right) .
$$

The cost function $J^{\varepsilon}$ is of class $C^{1}$, the adjoint problem associated to the problem (3.1) and the cost function $J^{\varepsilon}$ is defined as follows

$$
a^{\varepsilon}\left(w, p^{\varepsilon}\right)=-\partial_{u} J^{\varepsilon}\left(u^{\varepsilon}\right) w \quad \forall w \in \mathcal{V}^{\varepsilon},
$$

where $p^{\varepsilon}$ is the solution of this problem.

Suppose that $a^{\varepsilon}, \ell^{\varepsilon}$ and $J^{\varepsilon}$ are integrals over a domain $\Omega$. 
The domain $\Omega$ is split into two parts, a part $D$ containing the perturbation, and its complementary $\Omega_{0}=\Omega \backslash \bar{D}$. The perturbation can be located on the boundary of $\Omega$. For this reason, the domain $\bar{D}$ is not necessarily included in the domain $\Omega$.

The forms $a^{\varepsilon}, \ell^{\varepsilon}$, and the cost function $J^{\varepsilon}$ are decomposed in the following way:

$-a^{\varepsilon}=a_{\Omega_{0}}+a_{D}^{\varepsilon}$

$-\ell^{\varepsilon}=\ell_{\Omega_{0}}+\ell_{D}^{\varepsilon}$

$-J^{\varepsilon}=J_{\Omega_{0}}+J_{D}^{\varepsilon}$,

where $a_{\Omega_{0}}, l_{\Omega_{0}}$ et $J_{\Omega_{0}}$ are independent of $\varepsilon$.

The constants $\alpha$ and $M$ are positive constants used in respectively the minoration and the majoration of the bilinear forms.

Similarly, we consider

$-\mathcal{V}_{\Omega_{0}}$, the space consisting of functions of $\mathcal{V}^{\varepsilon}$ and $\mathcal{V}^{0}$ restricted to $\Omega_{0}$;

- $\mathcal{V}_{D}^{\varepsilon}$, the space consisting of functions of $\mathcal{V}^{\varepsilon}$ restricted to $D$;

- $\mathcal{V}_{D}^{0}$, the space consisting of functions of $\mathcal{V}^{0}$ restricted to $D$;

- $\mathcal{V}_{D, 0}^{\varepsilon}$, the subspace of $\mathcal{V}_{D}^{\varepsilon}$, with null trace on $\partial D$;

- $\mathcal{V}_{\partial D}$, the space of traces on $\partial D$ of $\mathcal{V}_{\Omega_{0}}$.

\subsection{Updating the direct solution}

In this section, we assume that $\mathcal{V}^{0} \subset \mathcal{V}^{\varepsilon}$. When the perturbation consists in inserting a hole in the domain with a Dirichlet condition on the boundary of the hole, this condition does not hold. This case will be considered in Section 3.2.

Let us consider $u_{D}^{\varepsilon}$, the local update of $u^{0}$ :

$$
\left\{\begin{array}{l}
\text { Find } u_{D}^{\varepsilon} \in \mathcal{V}_{D}^{\varepsilon} \quad \text { solution of } \\
a_{D}^{\varepsilon}\left(u_{D}^{\varepsilon}, v\right)=\ell_{D}^{\varepsilon}(v) \quad \forall v \in \mathcal{V}_{D, 0}^{\varepsilon}, \\
u_{D}^{\varepsilon}=u^{0} \text { on } \partial D
\end{array}\right.
$$

and $\tilde{u}^{\varepsilon}$ the update of $u^{0}$ is given by:

$$
\tilde{u}^{\varepsilon}= \begin{cases}u_{D}^{\varepsilon} & \text { in } D \\ u^{0} & \text { in } \Omega_{0}\end{cases}
$$

We assume the following hypothesis.

Hypothesis 3.1. There exist three positive constants $\eta, C$ and $C_{u}$ independent of $\varepsilon$ and a positive real valued function $f$ defined on $\mathbb{R}_{+}$such that

$$
\begin{gathered}
\lim _{\varepsilon \rightarrow 0} f(\varepsilon)=0, \\
\left\|J^{\varepsilon}(v)-J^{\varepsilon}(u)-\partial_{u} J^{\varepsilon}(u)(v-u)\right\|_{\mathcal{V}^{\varepsilon}} \leq C\|v-u\|_{\mathcal{V}^{\varepsilon}}^{2}, \forall v, u \in B\left(u^{0}, \eta\right), \\
\left\|u^{\varepsilon}-u^{0}\right\|_{\mathcal{V}_{\Omega_{0}}} \leq C_{u} f(\varepsilon), \\
\lim _{\varepsilon \rightarrow 0}\left\|p^{\varepsilon}-p^{0}\right\|_{\mathcal{V}^{\varepsilon}}=0 .
\end{gathered}
$$

Proposition 3.2. Under Hypothesis 3.1, we have

$$
\left\|u^{\varepsilon}-\tilde{u}^{\varepsilon}\right\|_{\mathcal{V} \varepsilon}=\mathcal{O}(f(\varepsilon)) .
$$


Proof. Hypothesis 3.1 tells us that

$$
\left\|u^{\varepsilon}-u^{0}\right\|_{\mathcal{V}_{\Omega_{0}}}=\mathcal{O}(f(\varepsilon)) .
$$

The trace properties allows us to write:

$$
\left\|u^{\varepsilon}-u^{0}\right\|_{\mathcal{V}_{\partial D}}=\mathcal{O}(f(\varepsilon)) .
$$

We can write it under the following form

$$
\left\|u^{\varepsilon}-u^{0}\right\|_{\mathcal{V}_{\partial D}}=\min _{\phi=u^{\varepsilon}-u^{0} \text { on } \partial D}\|\phi\|_{\mathcal{V}_{D}^{\varepsilon}} .
$$

As a consequence, we define $\varphi \in \mathcal{V}_{D}^{\varepsilon}$ as the minimum of $\|\varphi\|_{\mathcal{V}_{D}^{\varepsilon}}$ under the constraint $\varphi=u^{\varepsilon}-u^{0}$ on $\partial D$, then

$$
\|\varphi\|_{\mathcal{V}_{D}^{\varepsilon}}=\left\|u^{\varepsilon}-u^{0}\right\|_{\mathcal{V}_{\partial D}}=\mathcal{O}(f(\varepsilon)) .
$$

We have $\varphi-\left(u^{\varepsilon}-u_{D}^{\varepsilon}\right)=0$ on $\partial D$, we can use the coercivity of $a_{D}^{\varepsilon}$ :

$$
\begin{aligned}
\alpha\left\|\varphi-\left(u^{\varepsilon}-u_{D}^{\varepsilon}\right)\right\|_{\mathcal{V}_{D}^{\varepsilon}}^{2} & \leq a_{D}^{\varepsilon}\left(\varphi-\left(u^{\varepsilon}-u_{D}^{\varepsilon}\right), \varphi-\left(u^{\varepsilon}-u_{D}^{\varepsilon}\right)\right) \\
& \leq a_{D}^{\varepsilon}\left(\varphi, \varphi-\left(u^{\varepsilon}-u_{D}^{\varepsilon}\right)\right)-a_{D}^{\varepsilon}\left(u^{\varepsilon}, \varphi-\left(u^{\varepsilon}-u_{D}^{\varepsilon}\right)\right)+a_{D}^{\varepsilon}\left(u_{D}^{\varepsilon}, \varphi-\left(u^{\varepsilon}-u_{D}^{\varepsilon}\right)\right),
\end{aligned}
$$

and the continuity of the bilinear form $a_{D}^{\varepsilon}$ :

$$
\begin{aligned}
\alpha\left\|\varphi-\left(u^{\varepsilon}-u_{D}^{\varepsilon}\right)\right\|_{\mathcal{V}_{D}^{\varepsilon}}^{2} & \leq M\|\varphi\|_{\mathcal{V}_{D}}\left\|\varphi-\left(u^{\varepsilon}-u_{D}^{\varepsilon}\right)\right\|_{\mathcal{V}_{D}}-\ell_{D}^{\varepsilon}\left(\varphi-\left(u^{\varepsilon}-u_{D}^{\varepsilon}\right)\right)+\ell_{D}^{\varepsilon}\left(\varphi-\left(u^{\varepsilon}-u_{D}^{\varepsilon}\right)\right) \\
& \leq M\left\|u^{\varepsilon}-u^{0}\right\| \mathcal{V}_{\partial D}\left\|\varphi-\left(u^{\varepsilon}-u_{D}^{\varepsilon}\right)\right\|_{\mathcal{V}_{D}^{\varepsilon}} .
\end{aligned}
$$

Using $\|\varphi\|_{\mathcal{V}_{D}^{\varepsilon}}=\mathcal{O}(f(\varepsilon)),\left\|\varphi-\left(u^{\varepsilon}-u_{D}^{\varepsilon}\right)\right\|_{\mathcal{V}_{D}^{\varepsilon}}=\mathcal{O}(f(\varepsilon))$ and the triangular inequality, we obtain:

$$
\left\|u^{\varepsilon}-u_{D}^{\varepsilon}\right\|_{\mathcal{V}_{D}^{\varepsilon}}=\mathcal{O}(f(\varepsilon)) .
$$

With the equality

$$
\left\|u^{\varepsilon}-\tilde{u}^{\varepsilon}\right\|_{\mathcal{V}^{\varepsilon}}^{2}=\left\|u^{\varepsilon}-u^{0}\right\|_{\mathcal{V}_{\Omega_{0}}}^{2}+\left\|u^{\varepsilon}-u_{D}^{\varepsilon}\right\|_{\mathcal{V}_{D}^{\varepsilon}}^{2}
$$

we obtain the final result.

Theorem 3.3. Under Hypothesis 3.1, we have

$$
j(\varepsilon)-j(0)=\mathcal{L}^{\varepsilon}\left(\tilde{u}^{\varepsilon}, p^{0}\right)-\mathcal{L}^{0}\left(u^{0}, p^{0}\right)+o(f(\varepsilon)) .
$$

According to litterature $[10,11,23,25,26,30]$, the difference $\mathcal{L}^{\varepsilon}\left(\tilde{u}^{\varepsilon}, p^{0}\right)-\mathcal{L}^{0}\left(u^{0}, p^{0}\right)$ is of order $f(\varepsilon)$.

Proof. We just split this variation into two terms

$$
j(\varepsilon)-j(0)=\mathcal{L}^{\varepsilon}\left(u^{\varepsilon}, p^{0}\right)-\mathcal{L}^{0}\left(u^{0}, p^{0}\right)=\left[\mathcal{L}^{\varepsilon}\left(u^{\varepsilon}, p^{0}\right)-\mathcal{L}^{\varepsilon}\left(\tilde{u}^{\varepsilon}, p^{0}\right)\right]+\left[\mathcal{L}^{\varepsilon}\left(\tilde{u}^{\varepsilon}, p^{0}\right)-\mathcal{L}^{0}\left(u^{0}, p^{0}\right)\right],
$$

and prove that

$$
\mathcal{L}^{\varepsilon}\left(u^{\varepsilon}, p^{0}\right)-\mathcal{L}^{\varepsilon}\left(\tilde{u}^{\varepsilon}, p^{0}\right)=o(f(\varepsilon)) .
$$

Using Hypothesis 3.1, we have

$$
\begin{aligned}
\mathcal{L}^{\varepsilon}\left(u^{\varepsilon}, p^{0}\right)-\mathcal{L}^{\varepsilon}\left(\tilde{u}^{\varepsilon}, p^{0}\right) & =J^{\varepsilon}\left(u^{\varepsilon}\right)-J^{\varepsilon}\left(\tilde{u}^{\varepsilon}\right)+a^{\varepsilon}\left(u^{\varepsilon}, p^{0}\right)-a^{\varepsilon}\left(\tilde{u}^{\varepsilon}, p^{0}\right) \\
& =\partial_{u} J^{\varepsilon}\left(u^{\varepsilon}\right)\left(u^{\varepsilon}-\tilde{u}^{\varepsilon}\right)+o(f(\varepsilon))+a^{\varepsilon}\left(u^{\varepsilon}-\tilde{u}^{\varepsilon}, p^{0}\right),
\end{aligned}
$$

and with equation (3.2), we obtain

$$
\begin{aligned}
\mathcal{L}^{\varepsilon}\left(u^{\varepsilon}, p^{0}\right)-\mathcal{L}^{\varepsilon}\left(\tilde{u}^{\varepsilon}, p^{0}\right) & =-a^{\varepsilon}\left(u^{\varepsilon}-\tilde{u}^{\varepsilon}, p^{\varepsilon}\right)+a^{\varepsilon}\left(u^{\varepsilon}-\tilde{u}^{\varepsilon}, p^{0}\right)+o(f(\varepsilon)) \\
& =a^{\varepsilon}\left(u^{\varepsilon}-\tilde{u}^{\varepsilon}, p^{0}-p^{\varepsilon}\right)+o(f(\varepsilon)) .
\end{aligned}
$$

Using the continuity of $a^{\varepsilon}$, Hypothesis 3.1 and Proposition 3.2, we obtain the final result. 
Remark 3.4. Generally, for topological perturbations $\left\|p^{\varepsilon}-p^{0}\right\|_{\mathcal{V}^{\varepsilon}}=\mathcal{O}\left((f(\varepsilon))^{\frac{1}{2}}\right)$ and $j(\varepsilon)-j(0)=\mathcal{L}^{\varepsilon}\left(\tilde{u}^{\varepsilon}, p^{0}\right)-$ $\mathcal{L}^{0}\left(u^{0}, p^{0}\right)+\mathcal{O}\left((f(\varepsilon))^{\frac{3}{2}}\right)[30]$. We will see in the following section that if we update locally $u$ and $p$, the rest could be of order $\mathcal{O}\left(f(\varepsilon)^{2}\right)$.

\subsection{Updating the direct and adjoint solutions}

In this section $\mathcal{V}^{0}$ is not necessary a sub-space of $\mathcal{V}^{\varepsilon}$. This is the case when the perturbation is a hole of radius $\varepsilon$ with a Dirichlet boundary condition on its boundary. The definition of $\tilde{u}^{\varepsilon}$ remains unchanged and we update $p^{0}$ in the same way.

Let us consider $p_{D}^{\varepsilon}$ the local update of $p^{0}$

$$
\begin{cases}\text { Find } p_{D}^{\varepsilon} \in \mathcal{V}_{D}^{\varepsilon} \text { solution of } & \\ a_{D}^{\varepsilon}\left(w, p_{D}^{\varepsilon}\right)=-\partial_{u} J_{D}^{\varepsilon}\left(u_{D}^{\varepsilon}\right) w, & \forall w \in \mathcal{V}_{D, 0}^{\varepsilon}, \\ p_{D}^{\varepsilon}=p^{0} & \text { on } \partial D,\end{cases}
$$

and $\tilde{p}^{\varepsilon}$ the update of $p^{0}$ is given by:

$$
\tilde{p}^{\varepsilon}= \begin{cases}p_{D}^{\varepsilon} & \text { in } D \\ p^{0} & \text { in } \Omega_{0} .\end{cases}
$$

We assume the following hypothesis.

Hypothesis 3.5. There exist four positive constants $\eta, C, C_{u}$ and $C_{p}$ independent of $\varepsilon$ and a positive real valued function $f$ defined on $\mathbb{R}_{+}$such that

$$
\begin{aligned}
\lim _{\varepsilon \rightarrow 0} f(\varepsilon) & =0, \\
\left\|J^{\varepsilon}(v)-J^{\varepsilon}(u)-\partial_{u} J^{\varepsilon}(u)(v-u)\right\|_{\mathcal{V}^{\varepsilon}} & \leq C\|v-u\|_{\mathcal{V}^{\varepsilon}}^{2}, \forall v, u \in B\left(u^{0}, \eta\right), \\
\left\|u^{\varepsilon}-u^{0}\right\|_{\mathcal{V}_{\Omega_{0}}} & \leq C_{u} f(\varepsilon), \\
\left\|p^{\varepsilon}-p^{0}\right\|_{\mathcal{V}_{\Omega_{0}}} & \leq C_{p} f(\varepsilon) .
\end{aligned}
$$

Proposition 3.6. Under Hypothesis 3.5, we have

$$
\left\|p^{\varepsilon}-\tilde{p}^{\varepsilon}\right\|_{\mathcal{V} \varepsilon}=\mathcal{O}(f(\varepsilon)) .
$$

Proof. Hypothesis 3.5 tells us that

$$
\left\|p^{\varepsilon}-p^{0}\right\|_{\mathcal{\nu}_{\Omega_{0}}}=\mathcal{O}(f(\varepsilon))
$$

The trace properties allows us to write:

$$
\left\|p^{\varepsilon}-p^{0}\right\|_{\mathcal{V}_{\partial D}}=\mathcal{O}(f(\varepsilon))
$$

We can write it under the following form

$$
\left\|p^{\varepsilon}-p^{0}\right\| \mathcal{V}_{\partial D}=\min _{\phi=p^{\varepsilon}-p^{0} \text { on } \partial D}\|\phi\|_{\mathcal{V}_{D}^{\varepsilon}} .
$$

As a consequence, we define $\varphi \in \mathcal{V}_{D}^{\varepsilon}$ as the minimum of $\|\varphi\|_{\mathcal{V}_{D}^{\varepsilon}}$ under the constraint $\varphi=p^{\varepsilon}-p^{0}$ on $\partial D$, then

$$
\|\varphi\|_{\mathcal{V}_{D}^{\varepsilon}}=\left\|p^{\varepsilon}-p^{0}\right\|_{\mathcal{V}_{\partial D}}=\mathcal{O}(f(\varepsilon)) .
$$

We have $\varphi-\left(p^{\varepsilon}-p_{D}^{\varepsilon}\right)=0$ on $\partial D$, we can use the coercivity of $a_{D}^{\varepsilon}$ :

$$
\begin{aligned}
\alpha\left\|\varphi-\left(p^{\varepsilon}-p_{D}^{\varepsilon}\right)\right\|_{\mathcal{V}_{D}^{\varepsilon}}^{2} & \leq a_{D}^{\varepsilon}\left(\varphi-\left(p^{\varepsilon}-p_{D}^{\varepsilon}\right), \varphi-\left(p^{\varepsilon}-p_{D}^{\varepsilon}\right)\right) \\
& \leq a_{D}^{\varepsilon}\left(\varphi-\left(p^{\varepsilon}-p_{D}^{\varepsilon}\right), \varphi\right)-a_{D}^{\varepsilon}\left(\varphi-\left(p^{\varepsilon}-p_{D}^{\varepsilon}\right), p^{\varepsilon}\right)+a_{D}^{\varepsilon}\left(\varphi-\left(p^{\varepsilon}-p_{D}^{\varepsilon}\right), p_{D}^{\varepsilon}\right) .
\end{aligned}
$$


Equations (3.2) and (3.5) give us:

$$
\begin{aligned}
\alpha\left\|\varphi-\left(p^{\varepsilon}-p_{D}^{\varepsilon}\right)\right\|_{\mathcal{V}_{D}^{\varepsilon}}^{2} & \leq a_{D}^{\varepsilon}\left(\varphi-\left(p^{\varepsilon}-p_{D}^{\varepsilon}\right), \varphi\right)+\partial_{u} J_{D}^{\varepsilon}\left(u^{\varepsilon}\right)\left(\varphi-\left(p^{\varepsilon}-p_{D}^{\varepsilon}\right)\right)-\partial_{u} J_{D}^{\varepsilon}\left(u_{D}^{\varepsilon}\right)\left(\varphi-\left(p^{\varepsilon}-p_{D}^{\varepsilon}\right)\right) \\
& \leq a_{D}^{\varepsilon}\left(\varphi-\left(p^{\varepsilon}-p_{D}^{\varepsilon}\right), \varphi\right)+\partial_{u u} J_{D}^{\varepsilon}\left(u^{\varepsilon}\right)\left(\varphi-\left(p^{\varepsilon}-p_{D}^{\varepsilon}\right), u^{\varepsilon}-u_{D}^{\varepsilon}\right)+\left\|\varphi-\left(p^{\varepsilon}-p_{D}^{\varepsilon}\right)\right\|_{\mathcal{V}_{D}^{\varepsilon}} o(f(\varepsilon)) .
\end{aligned}
$$

The continuity of the bilinear form $a_{D}^{\varepsilon}$ and the continuity of the linear form $\partial_{u} J_{D}^{\varepsilon}$ lead to the following result:

$$
\begin{aligned}
\alpha\left\|\varphi-\left(p^{\varepsilon}-p_{D}^{\varepsilon}\right)\right\|_{\mathcal{V}_{D}^{\varepsilon}} & \leq M\left(\|\varphi\|_{\mathcal{V}_{D}^{\varepsilon}}+\left\|u^{\varepsilon}-u_{D}^{\varepsilon}\right\|_{\mathcal{V}_{D}^{\varepsilon}}+o(f(\varepsilon))\right) \\
& \leq M\left(\left\|p^{\varepsilon}-p^{0}\right\| \mathcal{V}_{\partial D}+\left\|u^{\varepsilon}-u_{D}^{\varepsilon}\right\|_{\mathcal{V}_{D}^{\varepsilon}}+o(f(\varepsilon))\right) .
\end{aligned}
$$

Thanks to $\left\|p^{\varepsilon}-p^{0}\right\|_{\mathcal{V}_{\partial D}}=\mathcal{O}(f(\varepsilon))$ and Proposition $3.2\left\|u^{\varepsilon}-u_{D}^{\varepsilon}\right\|_{\mathcal{V}_{D}^{\varepsilon}}=\mathcal{O}(f(\varepsilon))$, we have $\left\|\varphi-\left(p^{\varepsilon}-p_{D}^{\varepsilon}\right)\right\|_{\mathcal{V}_{D}^{\varepsilon}}=$ $\mathcal{O}(f(\varepsilon))$.

Using $\|\varphi\|_{\mathcal{V}_{D}^{\varepsilon}}=\mathcal{O}(f(\varepsilon)),\left\|\varphi-\left(p^{\varepsilon}-p_{D}^{\varepsilon}\right)\right\|_{\mathcal{V}_{D}^{\varepsilon}}=\mathcal{O}(f(\varepsilon))$ and the triangular inequality, we obtain:

$$
\left\|p^{\varepsilon}-p_{D}^{\varepsilon}\right\|_{\mathcal{V}_{D}^{\varepsilon}}=\mathcal{O}(f(\varepsilon)) .
$$

With the equality

$$
\left\|p^{\varepsilon}-\tilde{p}^{\varepsilon}\right\|_{\mathcal{V}^{\varepsilon}}^{2}=\left\|p^{\varepsilon}-p^{0}\right\|_{\mathcal{V}_{\Omega_{0}}}^{2}+\left\|p^{\varepsilon}-p_{D}^{\varepsilon}\right\|_{\mathcal{V}_{D}^{\varepsilon}}^{2}
$$

we obtain the final result.

Theorem 3.7. Under Hypothesis 3.5, we have

$$
j(\varepsilon)-j(0)=\mathcal{L}^{\varepsilon}\left(\tilde{u}^{\varepsilon}, \tilde{p}^{\varepsilon}\right)-\mathcal{L}^{0}\left(u^{0}, p^{0}\right)+\mathcal{O}\left(f(\varepsilon)^{2}\right) .
$$

According to litterature $[10,11,23,25,26,30]$, the difference $\mathcal{L}^{\varepsilon}\left(\tilde{u}^{\varepsilon}, \tilde{p}^{\varepsilon}\right)-\mathcal{L}^{0}\left(u^{0}, p^{0}\right)$ is of order $f(\varepsilon)$.

Proof. We just split this variation into two terms

$$
\begin{aligned}
j(\varepsilon)-j(0) & =\mathcal{L}^{\varepsilon}\left(u^{\varepsilon}, p^{\varepsilon}\right)-\mathcal{L}^{0}\left(u^{0}, p^{0}\right) \\
& =\left[\mathcal{L}^{\varepsilon}\left(u^{\varepsilon}, p^{\varepsilon}\right)-\mathcal{L}^{\varepsilon}\left(\tilde{u}^{\varepsilon}, p^{\varepsilon}\right)\right]+\left[\mathcal{L}^{\varepsilon}\left(\tilde{u}^{\varepsilon}, p^{\varepsilon}\right)-\mathcal{L}^{0}\left(u^{0}, p^{0}\right)\right],
\end{aligned}
$$

and prove that

$$
\mathcal{L}^{\varepsilon}\left(u^{\varepsilon}, p^{\varepsilon}\right)-\mathcal{L}^{\varepsilon}\left(\tilde{u}^{\varepsilon}, \tilde{p}^{\varepsilon}\right)=\mathcal{O}\left(f(\varepsilon)^{2}\right)
$$

Using Hypothesis 3.5, we have

$$
\begin{aligned}
\mathcal{L}^{\varepsilon}\left(u^{\varepsilon}, p^{\varepsilon}\right)-\mathcal{L}^{\varepsilon}\left(\tilde{u}^{\varepsilon}, \tilde{p}^{\varepsilon}\right) & =J^{\varepsilon}\left(u^{\varepsilon}\right)-J^{\varepsilon}\left(\tilde{u}^{\varepsilon}\right)+a^{\varepsilon}\left(u^{\varepsilon}, p^{\varepsilon}\right)-a^{\varepsilon}\left(\tilde{u}^{\varepsilon}, \tilde{p}^{\varepsilon}\right)-\ell^{\varepsilon}\left(p^{\varepsilon}\right)+\ell^{\varepsilon}\left(\tilde{p}^{\varepsilon}\right) \\
& =\partial_{u} J^{\varepsilon}\left(u^{\varepsilon}\right)\left(u^{\varepsilon}-\tilde{u}^{\varepsilon}\right)+\mathcal{O}\left(f(\varepsilon)^{2}\right)+a^{\varepsilon}\left(u^{\varepsilon}-\tilde{u}^{\varepsilon}, p^{\varepsilon}\right)+a^{\varepsilon}\left(\tilde{u}^{\varepsilon}, p^{\varepsilon}-\tilde{p}^{\varepsilon}\right)-\ell^{\varepsilon}\left(p^{\varepsilon}-\tilde{p}^{\varepsilon}\right)
\end{aligned}
$$

and with equations (3.1) and (3.2), we obtain

$$
\begin{aligned}
\mathcal{L}^{\varepsilon}\left(u^{\varepsilon}, p^{\varepsilon}\right)-\mathcal{L}^{\varepsilon}\left(\tilde{u}^{\varepsilon}, \tilde{p}^{\varepsilon}\right) & =a^{\varepsilon}\left(\tilde{u}^{\varepsilon}, p^{\varepsilon}-\tilde{p}^{\varepsilon}\right)-\ell^{\varepsilon}\left(p^{\varepsilon}-\tilde{p}^{\varepsilon}\right)+\mathcal{O}\left(f(\varepsilon)^{2}\right) \\
& =a^{\varepsilon}\left(\tilde{u}^{\varepsilon}-u^{\varepsilon}, p^{\varepsilon}-\tilde{p}^{\varepsilon}\right)+\mathcal{O}\left(f(\varepsilon)^{2}\right) .
\end{aligned}
$$

Using the continuity of $a^{\varepsilon}$, Propositions 3.2 and 3.6, we obtain the final result.

Remark 3.8. Updating $p$ is not necessary when $\mathcal{V}^{0} \subset \mathcal{V}^{\varepsilon}$, but according to Theorem 3.7, we obtain a better estimation of the variation of $j$ when $p$ is updated.

In the case of a Dirichlet condition on the boundary of the hole, $\mathcal{V}^{0}$ is not a subspace of $\mathcal{V}^{\varepsilon}$ and we need to update the adjoint. 


\subsection{Examples}

\subsubsection{Examples in literature}

The most important hypothesis is

$$
\left\|u^{\varepsilon}-u^{0}\right\|_{\mathcal{V}_{\Omega_{0}}}=\mathcal{O}(f(\varepsilon)) .
$$

This assumption (3.7) is generally satisfied. See

- the elasticity case [23];

- the Poisson equation [25];

- the Navier-Stokes equation [26];

- the Helmholtz equation [10].

These results have been obtained in the frame of the domain truncation method.

\subsubsection{The capacity case}

This section presents an application of the previous results to the capacity problem. Let $\Omega \in \mathbb{R}^{2}$ be a domain.

$$
\begin{cases}\Delta u^{0}=0 & \text { in } \Omega, \\ u^{0}=1 & \text { on } \partial \Omega .\end{cases}
$$

This equation implies that $u^{0}=1$ in $\Omega$ is solution.

The cost function is the following

$$
J^{\varepsilon}(v)=\frac{1}{2} \int_{\Omega_{\varepsilon}}|\nabla v|^{2} \mathrm{~d} x, \quad \forall v \in V .
$$

Let $B\left(x_{0}, \varepsilon\right)$ be a hole in the domain $\Omega$, the perturbed domain is $\Omega_{\varepsilon}$.

$$
\begin{cases}\Delta u^{\varepsilon}=0 & \text { in } \Omega_{\varepsilon}, \\ u^{\varepsilon}=0 & \text { on } \partial B\left(x_{0}, \varepsilon\right), \\ u^{\varepsilon}=1 & \text { on } \partial \Omega .\end{cases}
$$

Let $D$ be equal to $B\left(x_{0}, R\right) \backslash B\left(x_{0}, \varepsilon\right)$ with $R>\varepsilon$ and $B(x, R) \subset \Omega$.

The update of $u^{0}$ is defined by

$$
\tilde{u}^{\varepsilon}= \begin{cases}u_{D}^{\varepsilon} & \text { in } D, \\ u^{0} & \text { in } \Omega \backslash D .\end{cases}
$$

where $u_{D}^{\varepsilon}$ is the solution of the following equation

$$
\begin{cases}\Delta u_{D}^{\varepsilon}=0 & \text { in } D, \\ u_{D}^{\varepsilon}=0 & \text { on } \partial B\left(x_{0}, \varepsilon\right), \\ u_{D}^{\varepsilon}=1 & \text { on } \partial B\left(x_{0}, R\right) .\end{cases}
$$

The hypothesis $\left\|u^{\varepsilon}-u^{0}\right\|_{\mathcal{V}_{\Omega_{0}}}=\mathcal{O}(f(\varepsilon))$ is satisfied [30].

The elementary solution of the Laplace equation in two dimensions is $-\frac{\ln (r)}{2 \pi}$ where $r=\left|x-x_{0}\right|$. The update is

$$
\tilde{u}^{\varepsilon}= \begin{cases}\frac{\ln \left(\left|x-x_{0}\right|\right)-\ln (\varepsilon)}{\ln (R)-\ln (\varepsilon)} & \text { in } D \\ 1 & \text { in } \Omega \backslash D .\end{cases}
$$


Let $\Gamma$ be the outer boundary of the domain $D$ and $n$ the normal oriented towards the exterior on the boundary. With the approximation $\tilde{u}^{\varepsilon}$, the cost function (3.9) can be written as follows

$$
J^{\varepsilon}\left(\tilde{u}^{\varepsilon}\right)=\pi \int_{\Gamma} \frac{\partial \tilde{u}^{\varepsilon}}{\partial n} \tilde{u}^{\varepsilon} r \mathrm{~d} r .
$$

Taking into account Theorem 3.3 we obtain

$$
j(\varepsilon)-j(0)=J^{\varepsilon}\left(u^{\varepsilon}\right)-J^{0}\left(u^{0}\right)=J^{\varepsilon}\left(\tilde{u}^{\varepsilon}\right)-J^{0}\left(u^{0}\right)+o(f(\varepsilon)),
$$

and given the update definition (3.13), we have

$$
\begin{aligned}
J^{\varepsilon}\left(\tilde{u}^{\varepsilon}\right)-J^{0}\left(u^{0}\right) & =\pi \int_{\varepsilon}^{R}\left|\nabla u^{\varepsilon}\right|^{2} r \mathrm{~d} r \\
\Leftrightarrow J^{\varepsilon}\left(\tilde{u}^{\varepsilon}\right)-J^{0}\left(u^{0}\right) & =\frac{\pi}{(\ln (R)-\ln (\varepsilon))^{2}} \int_{r=R} \frac{1}{r}(\ln (r)-\ln (\varepsilon)) r \mathrm{~d} r \\
\Leftrightarrow J^{\varepsilon}\left(\tilde{u}^{\varepsilon}\right)-J^{0}\left(u^{0}\right) & =\frac{\pi}{(\ln (R)-\ln (\varepsilon))} .
\end{aligned}
$$

The final result is

$$
j(\varepsilon)-j(0)=J^{\varepsilon}\left(u^{\varepsilon}\right)-J^{0}\left(u^{0}\right)=\frac{-\pi}{\ln (\varepsilon)}+o\left(\frac{1}{\ln (\varepsilon)}\right) .
$$

This result is exactly the same as the one obtained in [30].

\section{Approximation of a perturbed solution}

In this section, we assume that $\mathcal{V}^{0} \subset \mathcal{V}^{\varepsilon}$. The aim of the previous section was to estimate the variation of a cost function with respect to a local perturbation. In this part, the vault method is used to construct an approximation of the perturbed solution $u$.

We assume the following hypothesis.

Hypothesis 4.1. There exists a constant $C_{u}$ independent of $\varepsilon$ and a positive real valued function $f$ defined on $\mathbb{R}_{+}$such that

$$
\begin{gathered}
\lim _{\varepsilon \rightarrow 0} f(\varepsilon)=0, \\
\left\|u^{\varepsilon}-u^{0}\right\|_{\mathcal{V}_{\Omega_{0}}} \leq C_{u} f(\varepsilon), \\
\left\|a_{D}^{\varepsilon}-a_{D}^{0}\right\|_{\mathcal{L}^{2}\left(\mathcal{V}_{D}^{\varepsilon}\right)}=\mathcal{O}(f(\varepsilon)) .
\end{gathered}
$$

The norm $\|\cdot\|_{\mathcal{L}^{2}\left(\mathcal{V}_{D}^{\varepsilon}\right)}$ means the norm of the bilinear form.

Proposition 4.2. Under Hypothesis 4.1, we have

$$
\begin{gathered}
\left\|u^{\varepsilon}-\tilde{u}^{\varepsilon}\right\|_{\mathcal{V}^{\varepsilon}}=\mathcal{O}(f(\varepsilon)), \\
\left\|a^{\varepsilon}-a^{0}\right\|_{\mathcal{L}^{2}\left(\mathcal{V}^{\varepsilon}\right)}=\mathcal{O}(f(\varepsilon)) .
\end{gathered}
$$

Proof. Thanks to Proposition 3.2, we have

$$
\left\|u^{\varepsilon}-\tilde{u}^{\varepsilon}\right\|_{\mathcal{V}^{\varepsilon}}=\mathcal{O}(f(\varepsilon)) .
$$

As $a^{0}=a_{\Omega_{0}}+a_{D}^{0}$ and $a^{\varepsilon}=a_{\Omega_{0}}+a_{D}^{\varepsilon}$, we have

$$
\left\|a^{\varepsilon}-a^{0}\right\|_{\mathcal{L}^{2}\left(\mathcal{V}^{\varepsilon}\right)}=\mathcal{O}(f(\varepsilon)) .
$$


A singular local perturbation has two effects: a local high frequency effect and a global low frequency effect. The local effect is caught by $\tilde{u}^{\varepsilon}$. In the previous section, the global effect is taken into account by the adjoint. In this section, we are considering the variation of the solution, the adjoint cannot be used, and we need to solve an auxiliary problem in order to calculate the global low frequency variation.

Let us consider $\delta u^{\varepsilon}$ the solution of

$$
\left\{\begin{array}{l}
\text { Find } \delta u^{\varepsilon} \in \mathcal{V}^{0} \text { solution of } \\
a^{0}\left(\delta u^{\varepsilon}, v\right)=a^{\varepsilon}\left(u^{\varepsilon}-\tilde{u}^{\varepsilon}, v\right), \quad \forall v \in \mathcal{V}^{0} .
\end{array}\right.
$$

The approximation of $u^{\varepsilon}$ with the numerical vault is noted $\tilde{\tilde{u}}^{\varepsilon}$ and defined as follows

$$
\tilde{\tilde{u}}^{\varepsilon}=\tilde{u}^{\varepsilon}+\delta u^{\varepsilon} .
$$

Proposition 4.3. Under Hypothesis 4.1, we have

$$
\left\|\delta u^{\varepsilon}\right\|_{\mathcal{V}^{0}}=\mathcal{O}(f(\varepsilon)) .
$$

Proof. Thanks to the coercivity of $a^{0}$, we have

$$
\alpha\left\|\delta u^{\varepsilon}\right\|_{\mathcal{V}^{0}}^{2} \leq a^{0}\left(\delta u^{\varepsilon}, \delta u^{\varepsilon}\right)=a^{\varepsilon}\left(u^{\varepsilon}-\tilde{u}^{\varepsilon}, \delta u^{\varepsilon}\right) .
$$

Thanks to the continuity of $a^{\varepsilon}$, we have

$$
a^{\varepsilon}\left(u^{\varepsilon}-\tilde{u}^{\varepsilon}, \delta u^{\varepsilon}\right) \leq M\left\|u^{\varepsilon}-\tilde{u}^{\varepsilon}\right\|_{\mathcal{V}^{\varepsilon}}\left\|\delta u^{\varepsilon}\right\|_{\mathcal{V}^{0}} .
$$

Hypothesis 4.1 and Proposition 4.2 allow us to conclude.

Theorem 4.4. Under Hypothesis 4.1 and with the definition of $\tilde{\tilde{u}}^{\varepsilon}$, we have

$$
\left\|u^{\varepsilon}-\tilde{\tilde{u}}^{\varepsilon}\right\|_{\mathcal{V}^{\varepsilon}}=\mathcal{O}\left((f(\varepsilon))^{2}\right) .
$$

Proof. Thanks to the coercivity of $a^{\varepsilon}$, we have

$$
\alpha\left\|u^{\varepsilon}-\tilde{\tilde{u}}^{\varepsilon}\right\|_{\mathcal{V}^{\varepsilon}}^{2} \leq a^{\varepsilon}\left(u^{\varepsilon}-\tilde{\tilde{u}}^{\varepsilon}, u^{\varepsilon}-\tilde{\tilde{u}}^{\varepsilon}\right)=a^{\varepsilon}\left(u^{\varepsilon}-\tilde{u}^{\varepsilon}, u^{\varepsilon}-\tilde{\tilde{u}}^{\varepsilon}\right)-a^{\varepsilon}\left(\delta u^{\varepsilon}, u^{\varepsilon}-\tilde{\tilde{u}}^{\varepsilon}\right) .
$$

Thanks to the definition of $\tilde{\tilde{u}}^{\varepsilon}$, we have

$$
\alpha\left\|u^{\varepsilon}-\tilde{\tilde{u}}^{\varepsilon}\right\|_{\mathcal{V}^{\varepsilon}}^{2} \leq a^{0}\left(\delta u^{\varepsilon}, u^{\varepsilon}-\tilde{\tilde{u}}^{\varepsilon}\right)-a^{\varepsilon}\left(\delta u^{\varepsilon}, u^{\varepsilon}-\tilde{\tilde{u}}^{\varepsilon}\right) .
$$

Thanks to Proposition 4.2, we have

$$
\left\|a^{\varepsilon}-a^{0}\right\|_{\mathcal{L}^{2}\left(\mathcal{V}^{\varepsilon}\right)}=\mathcal{O}(f(\varepsilon))
$$

so

$$
\left|a^{0}\left(\delta u^{\varepsilon}, u^{\varepsilon}-\tilde{\tilde{u}}^{\varepsilon}\right)-a^{\varepsilon}\left(\delta u^{\varepsilon}, u^{\varepsilon}-\tilde{\tilde{u}}^{\varepsilon}\right)\right| \leq C f(\varepsilon)\left\|\delta u^{\varepsilon}\right\|_{\mathcal{V}^{0}}\left\|u^{\varepsilon}-\tilde{\tilde{u}}^{\varepsilon}\right\|_{\mathcal{V}^{\varepsilon}} .
$$

Thanks to Proposition 4.3, we have

$$
\left\|\delta u^{\varepsilon}\right\|_{\mathcal{V}^{0}}=\mathcal{O}(f(\varepsilon))
$$

This implies that

$$
\left\|u^{\varepsilon}-\tilde{\tilde{u}}^{\varepsilon}\right\|_{\mathcal{V}^{\varepsilon}}=\mathcal{O}\left((f(\varepsilon))^{2}\right) .
$$

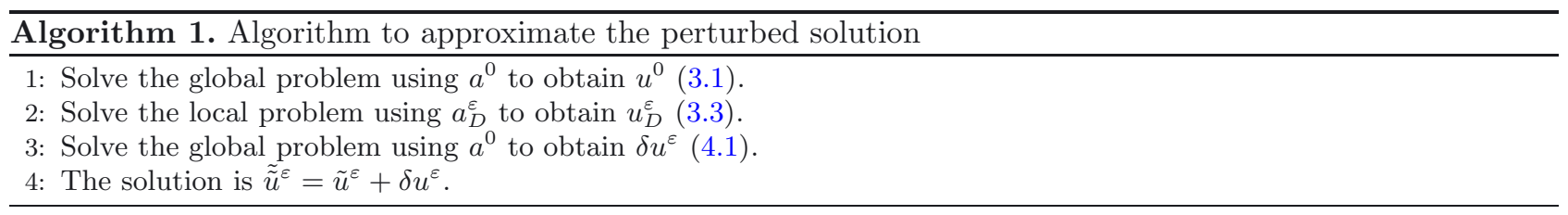


We solved a local problem and two global problems at $\varepsilon=0$. The proposed method is interesting when the problem is easy to solve at $\varepsilon=0$. This the case when the initial problem can be solved using spectral methods with constant coefficient.

It is also interesting when the matrix arising from $a^{0}$ is factorized. In the case of iterative method, when the same matrix is used to solve many problems, it is possible to build efficient preconditioners.

This method can be considered by researchers who do not use the adjoint method $[2,3,6,7,19,20]$. The low frequency effect can be taken into account by solving several time the same initial systems.

\section{Application to topological and CONTINUOUs VARIATions}

\subsection{Problem statement}

The aim of this section is to approximate the variation of a cost function in the following cases: topological pertubation, mesh perturbation, continuous variation of stiffness perturbation.

We model an experiment as follows: the domain $\Omega$ is a rectangle filled with an elastic material, the left vertical side is fixed and a vertical force of intensity $\mu$ in the downward direction is applied on the middle of the right vertical side, see Figure 1. The other sides are free.

Let $\Omega$ be a rectangular bounded domain of $\mathbb{R}^{2}$ and $\Gamma$ be its boundary, composed of two parts $\Gamma_{1}$ and $\Gamma_{2}$. The considered problem has Dirichlet boundary conditions on $\Gamma_{1}$ and Neumann boundary conditions on $\Gamma_{2}$. The rectangle is subject to a displacement $u$ solution of the following equation:

$$
\begin{cases}-\nabla \cdot \sigma(u)=0 & \text { in } \Omega, \\ u=0 & \text { on } \Gamma_{1}, \\ \sigma(u) n=g & \text { on } \Gamma_{2},\end{cases}
$$

with

$$
\begin{aligned}
\phi(u) & =\frac{1}{2}\left(D u+D u^{T}\right), \\
\sigma(u) & =h H_{0} \phi(u),
\end{aligned}
$$

where $H_{0}$ is the Hooke tensor and $h(x)=1$ and $\rho$ represents the material presence or absence.

The optimization problem consists in minimizing the following cost function

$$
j(h)=\int_{\Gamma_{2}} g \cdot u \mathrm{~d} x
$$

with the following constraint

where $C$ is the material quantity to retain.

$$
\int_{\Omega} h(x) \mathrm{d} x=C
$$

For each element, the function $h$ is modified, then the exact variation is computed using the cost function (5.2) with $u^{0}$ the solution of equation (5.1) and $u^{\varepsilon}$ the solution of

$$
\begin{cases}-\nabla \cdot \sigma^{\varepsilon}\left(u^{\varepsilon}\right)=0 & \text { in } \Omega^{\varepsilon}, \\ u^{\varepsilon}=0 & \text { on } \Gamma_{1}, \\ \sigma^{\varepsilon}\left(u^{\varepsilon}\right) n=g & \text { on } \Gamma_{2} .\end{cases}
$$

For each element, the numerical vault variation is computed using Theorem 3.7 which is equivalent to

$$
j\left(h^{\varepsilon}\right)-j(h)=\int_{\Gamma_{2}}\left(g \cdot \tilde{u}^{\varepsilon}-g \cdot u\right) \mathrm{d} x-\int_{\Omega^{\varepsilon}} \sigma\left(\tilde{u}^{\varepsilon}\right) \cdot \nabla\left(\tilde{u}^{\varepsilon}\right) \mathrm{d} x+\int_{\Omega} \sigma(u) \cdot \nabla(u) \mathrm{d} x,
$$

with

$$
\tilde{u}^{\varepsilon}= \begin{cases}u_{D}^{\varepsilon} & \text { in } D \\ u^{0} & \text { in } \Omega_{0}\end{cases}
$$



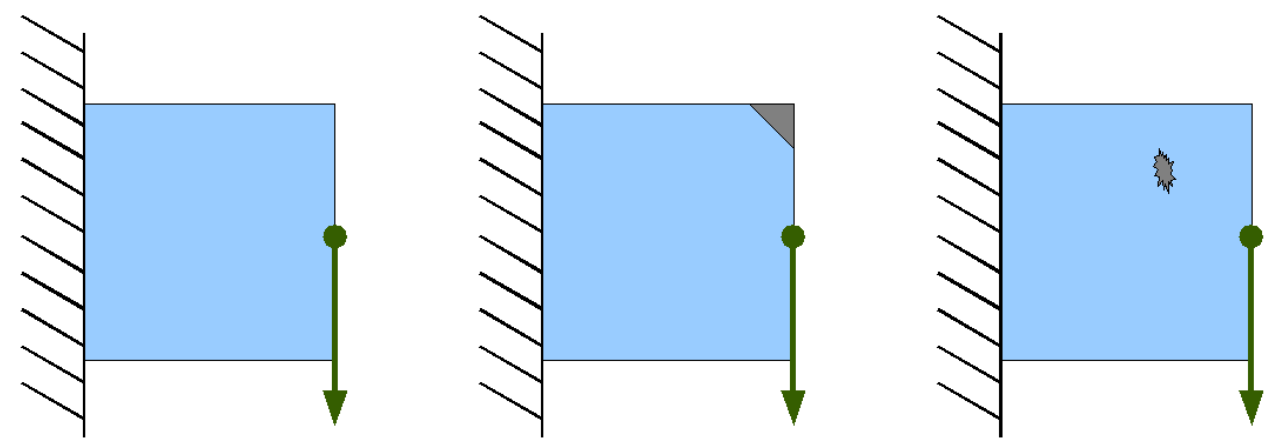

Figure 1. From left to right, a model without perturbation, two models with perturbations.

TABLE 1. Topological perturbation: comparative results of the variations obtained with the adjoint method and with the vault method.

\begin{tabular}{|c|c|c|c|c|c|c|c|}
\cline { 3 - 8 } \multicolumn{1}{c|}{} & \multicolumn{2}{c|}{$L^{1}$-norm } & \multicolumn{2}{c|}{$L^{2}$-norm } & \multicolumn{2}{c|}{$L^{\infty}$-norm } \\
\cline { 2 - 8 } \multicolumn{1}{c|}{} & Method & Difference & Relative err. & Difference & Relative err. & Difference & Relative err. \\
\hline \multirow{2}{*}{$\begin{array}{c}\text { Mesh } \\
516\end{array}$} & Adjoint & $5.64 \times 10^{-5}$ & 0.4191 & $9.53 \times 10^{-6}$ & 0.6729 & $7.10 \times 10^{-6}$ & 0.8012 \\
\cline { 2 - 8 } & Vault & $5.64 \times 10^{-6}$ & 0.0419 & $1.02 \times 10^{-6}$ & 0.0718 & $8.41 \times 10^{-7}$ & 0.0948 \\
\hline Mesh & Adjoint & $4.99 \times 10^{-5}$ & 0.3843 & $4.59 \times 10^{-6}$ & 0.6512 & $3.07 \times 10^{-6}$ & 0.8084 \\
\cline { 2 - 8 } 2064 & Vault & $3.21 \times 10^{-6}$ & 0.0247 & $5.02 \times 10^{-7}$ & 0.0711 & $3.88 \times 10^{-7}$ & 0.1023 \\
\hline Mesh & Adjoint & $4.50 \times 10^{-5}$ & 0.3587 & $2.01 \times 10^{-6}$ & 0.6141 & $1.18 \times 10^{-6}$ & 0.8147 \\
8256 & Vault & $2.34 \times 10^{-6}$ & 0.0186 & $2.15 \times 10^{-7}$ & 0.0660 & $1.50 \times 10^{-7}$ & 0.1035 \\
\hline
\end{tabular}

where $u_{D}^{\varepsilon}$ is the solution of the following equation

$$
\begin{cases}-\nabla \cdot \sigma^{\varepsilon}\left(u_{D}^{\varepsilon}\right)=0 & \text { in } D, \\ u_{D}^{\varepsilon}=0 & \text { on } \Gamma_{1}, \\ \sigma^{\varepsilon}\left(u_{D}^{\varepsilon}\right) n=g & \text { on } \Gamma_{2} .\end{cases}
$$

Remark 5.1. In this particular problem, the adjoint solution $p$ is equal to $-u$, so $u$ and $p$ have opposite updates.

\subsection{Numerical results}

In this section, the domain $D$ is made of four layers of elements around the perturbed element. The first experiment is relative to topological perturbation, the aim being to approximate the variation of the cost function when an element is removed. The actual variation of the cost function, the estimations obtained with the adjoint method and with the numerical vault are computed on three different meshes made of 516, 2064 and 8256 elements. The deletion of each mesh element is considered. Figure 2 shows the variation of the cost function for each element when this element is deleted. In Table 1, the differences in the $L^{1}, L^{2}$ and $L^{\infty}$ norms and the relative errors in the $L^{1}, L^{2}$ and $L^{\infty}$ norms are given for all the results obtained with the numerical vault and with the adjoint method. One can note that the results obtained with the numerical vault are nearer to the actual variation than the estimations obtained with the adjoint method.

The second experiment is relative to mesh perturbation, the aim being to approximate the variation of the cost function when the mesh is modified. To perform the mesh modifications, at each node, one node is moved towards the node on the left in the first layer of elements. The nodes on the boundary of the domain are not modified. The actual variation of the cost function, estimations obtained with the adjoint method and with 

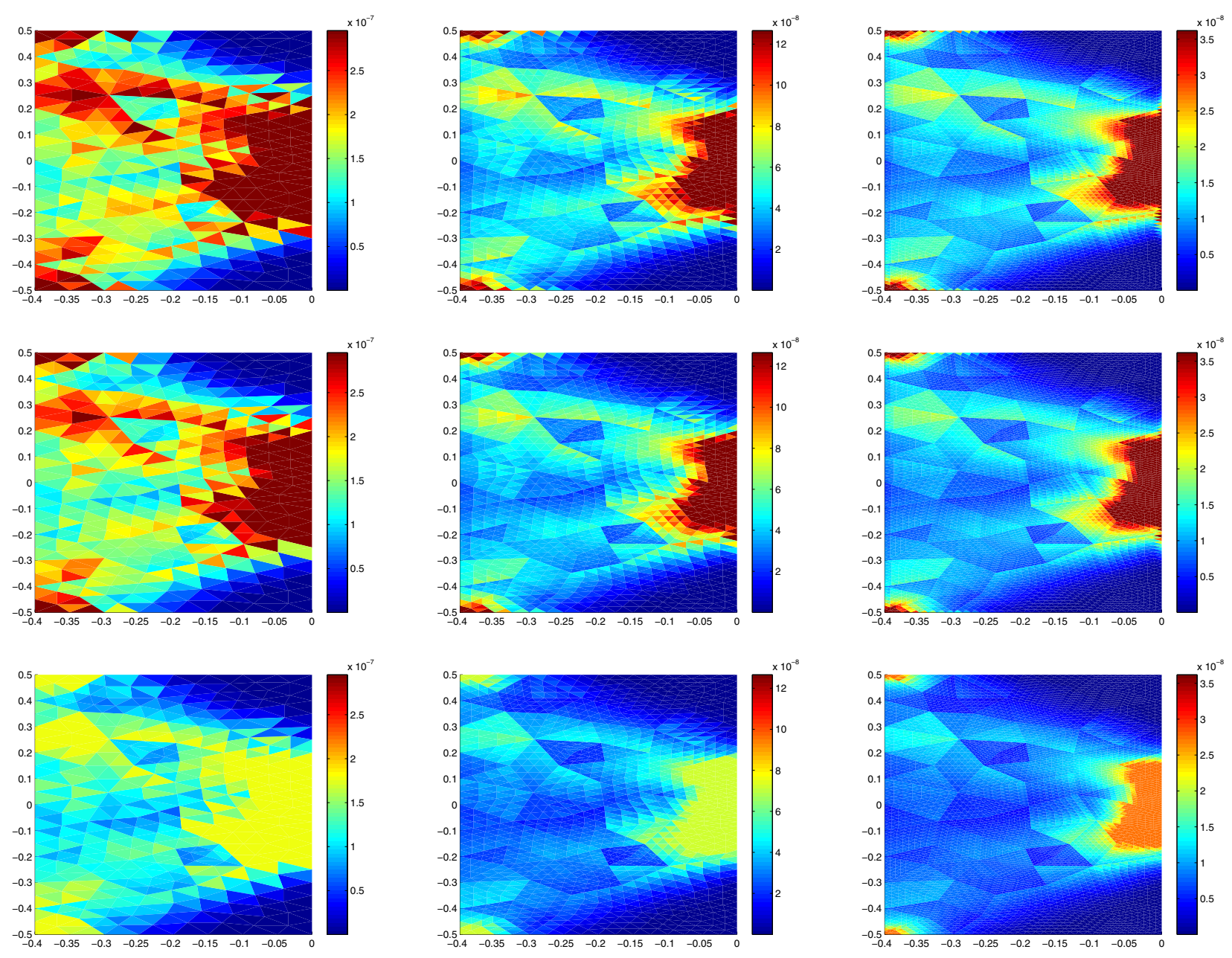

FiguRE 2. Topological perturbation: from left to right, mesh with 516, 2064 and 8256 elements. On the top row, actual variation, on the middle row, the estimation obtained with the numerical vault and on the bottom row, the estimation obtained with the adjoint method.

the numerical vault are computed on a mesh made of 2064 elements. Figure 3 shows the variation of the cost function for each node when this node is moved, the most interesting points are the legends of the plots. In Table 2, the differences in the $L^{1}, L^{2}$ and $L^{\infty}$ norms and the relative errors in the $L^{1}, L^{2}$ and $L^{\infty}$ norms are given for all the results obtained with the numerical vault and with the adjoint method. One can note that the numerical vault outperforms the adjoint method.

The last experiments concern continuous variations of stiffness perturbations. Figures 4 and 5 present different experiments which aim at comparing the estimations obtained by the vault method and by the adjoint method with the actual variation. The mesh is made of 8256 elements. For each experiment, the locations of the perturbations are given on the left image and the associated graphs are on the right. In the curves, the abscissa is the inhomogeneity stiffness varying from 0 to 2.5 with the material stiffness equal to 1 and the ordinate is the variation of the cost function. Different cases are considered by varying the location, the number and the size of the perturbations. 
TABLE 2. Mesh perturbation: comparative results of the variations obtained with the adjoint method and with the vault method.

\begin{tabular}{|c|c|c|c|c|c|c|}
\cline { 2 - 7 } \multicolumn{1}{c|}{} & \multicolumn{2}{c|}{$L^{1}$-norm } & \multicolumn{2}{c|}{$L^{2}$-norm } & \multicolumn{2}{c|}{$L^{\infty}$-norm } \\
\hline Method & Difference & Relative err. & Difference & Relative err. & Difference & Relative err. \\
\hline Adjoint & $1.75 \times 10^{-3}$ & 953.17 & $9.7249 \times 10^{-5}$ & 463.06 & $3.32 \times 10^{-5}$ & 367.67 \\
\hline Vault & $2.00 \times 10^{-7}$ & 0.1091 & $2.0570 \times 10^{-8}$ & 0.0979 & $5.41 \times 10^{-9}$ & 0.0599 \\
\hline
\end{tabular}
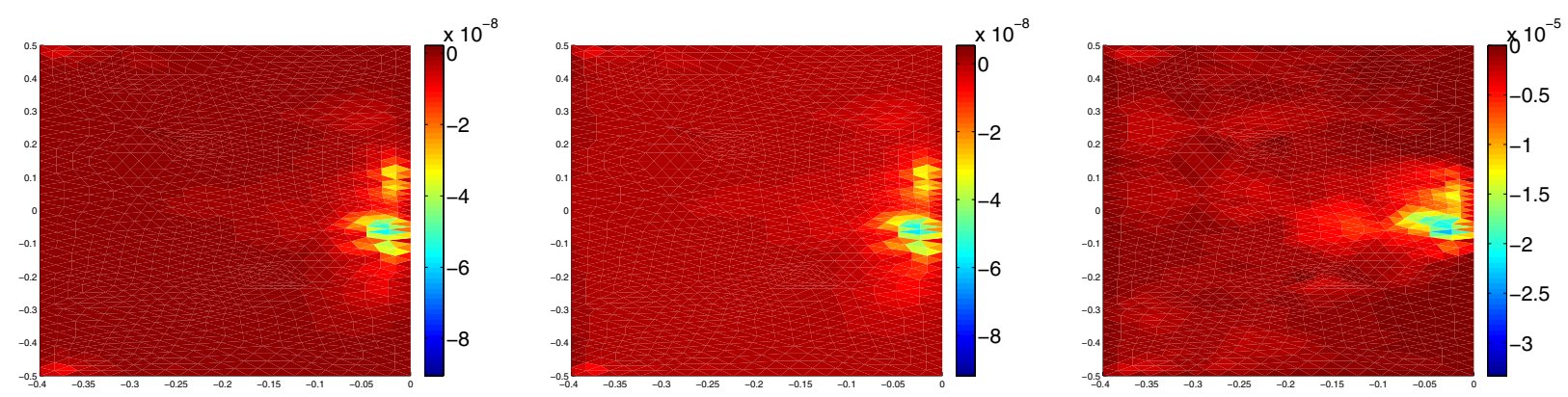

FiguRE 3. Mesh perturbation: from left to right, actual variation, the estimations obtained with the numerical vault and with the adjoint method.

In Figure 4, the beam has an homogeneous material property, a rectangular shape and some perturbations introduced. The perturbations are inside the beam or at the extremity or at random locations with two different sizes. In Figure 5, the beam has a predefined shape. In each graph, the abscissa is the material property of the perturbation and the ordinate is the computed result of the cost function with the different methods. The legend is the following: blue-circle for the exact variation, red-asterisk for the estimation obtained by numerical vault and green-square for the estimation obtained by the adjoint method. One can note that the estimation by the adjoint method is an approximation of first order and the vault method is more precise.

\section{Elastography}

\subsection{Problem statement}

The aim of this section is to detect small inclusions in elastography $[8,9,21,22,32,33]$ which is a technique giving the directional displacement of a material under motion. This medical imaging technique relies on the fact that elastic properties of tissues provide medical information that allows to detect or classify tumors which tend to be stiffer than the surrounding soft tissue.

This imaging experiment is only an example, other applications such localization of conductivity inhomogeneities can also be considered $[4,5]$.

We model an experiment as follows: the domain $\Omega$ is a rectangle filled with an elastic material, the left vertical side is fixed and the right vertical side is moved by a small amount $\mu$ in the left direction, see Figure 6 . The other sides are free. In order to ensure uniqueness of the resulting displacement, we also prevent vertical translations.

Let $\Omega$ be a rectangular bounded domain of $\mathbb{R}^{2}$ and $\Gamma$ be its boundary, composed of two parts $\Gamma_{1}$ and $\Gamma_{2}$. The points of the rectangle are submitted to a horizontal displacement $u$ solution of the following equation:

$$
\begin{cases}-\nabla \cdot \sigma(u)=0 & \text { in } \Omega, \\ u=0 & \text { on } \Gamma_{1}, \\ \sigma(u) n=\mu & \text { on } \Gamma_{2},\end{cases}
$$



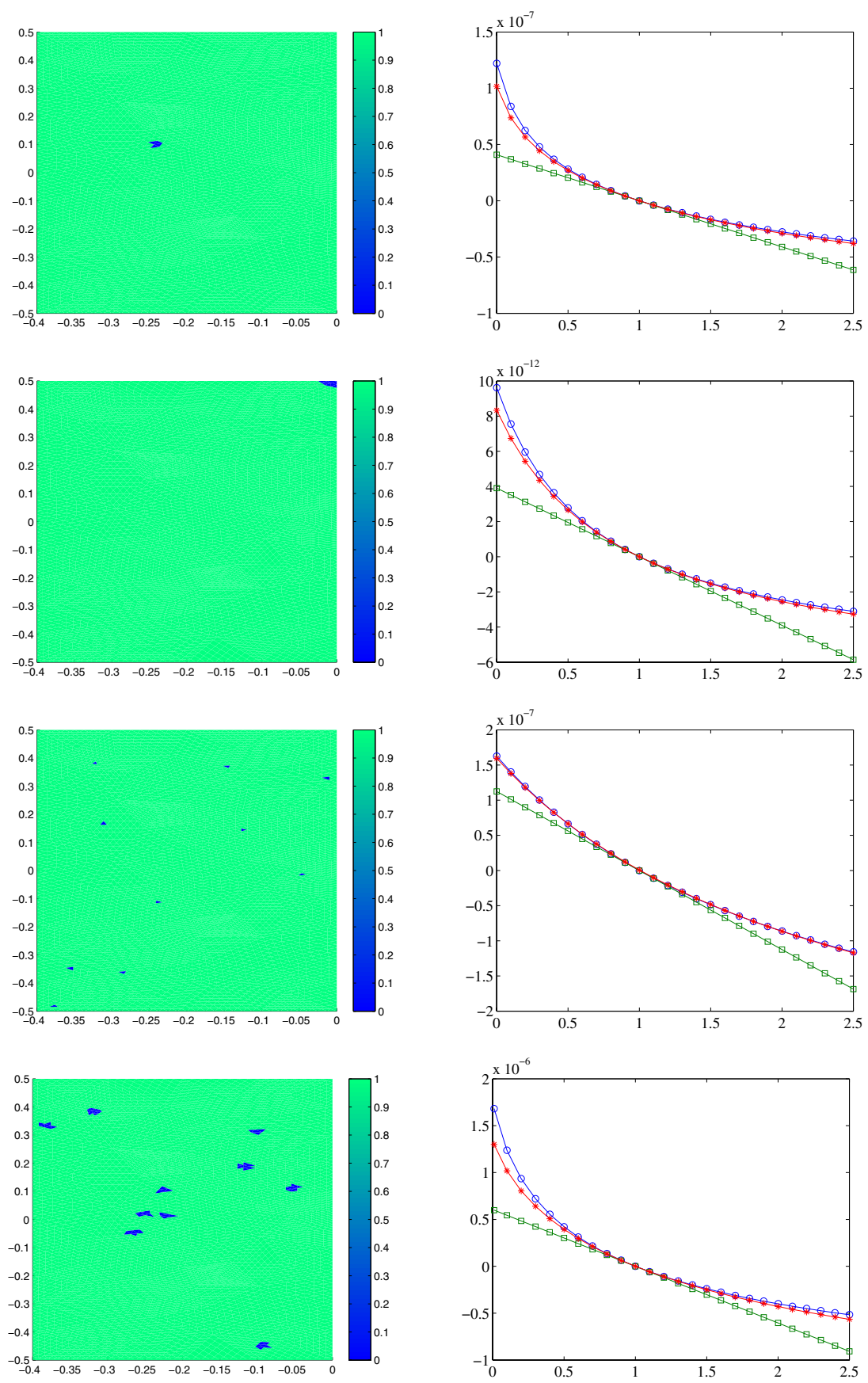

FiguRE 4. From left to right, the perturbations and the associated curves with blue-circle for the exact variation, red-asterisk for the estimation obtained by the numerical vault and greensquare for the estimation obtained by the adjoint method. The abscissa is the inhomogeneity stiffness varying from 0 to 2.5 with the material stiffness equal to 1 and the ordinate is the variation of the cost function. 

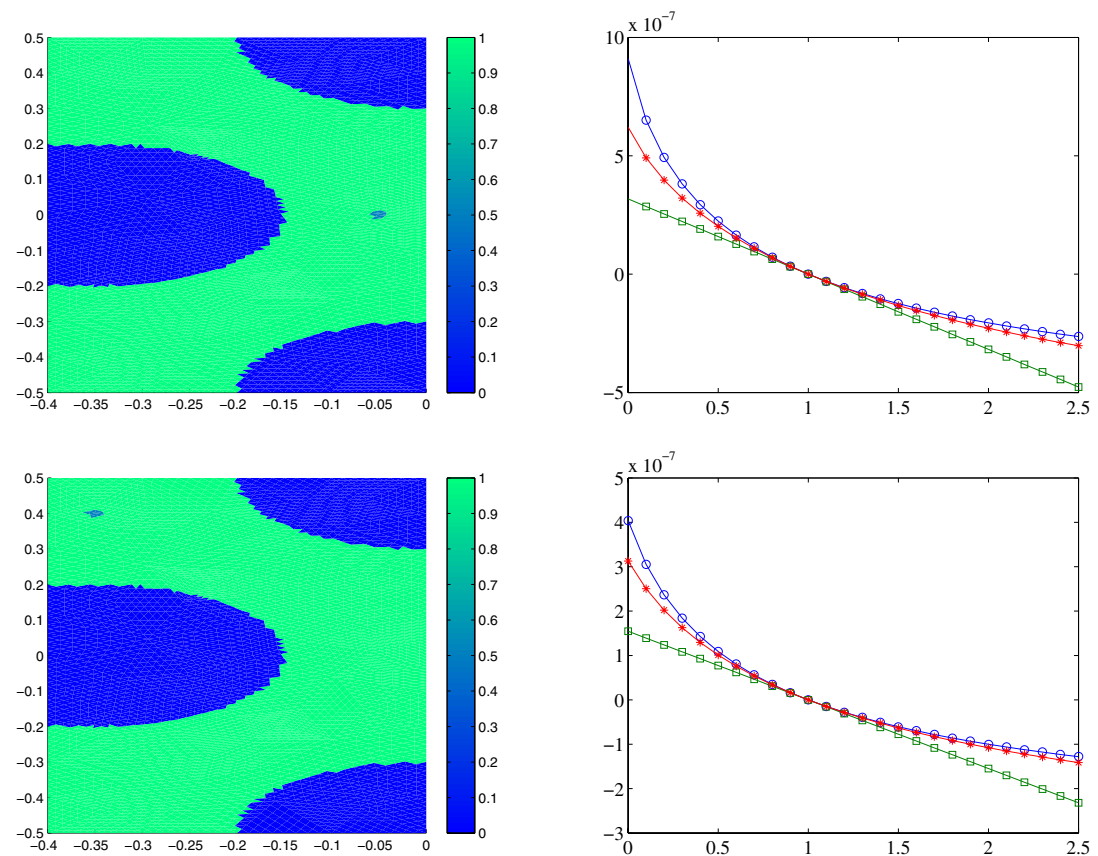

FiguRE 5. From left to right, the perturbations and the associated curves with blue-circle for the exact variation, red-asterisk for the estimation obtained by the numerical vault and greensquare for the estimation obtained by the adjoint method. The abscissa is the inhomogeneity stiffness varying from 0 to 2.5 with the material stiffness equal to 1 and the ordinate is the variation of the cost function.
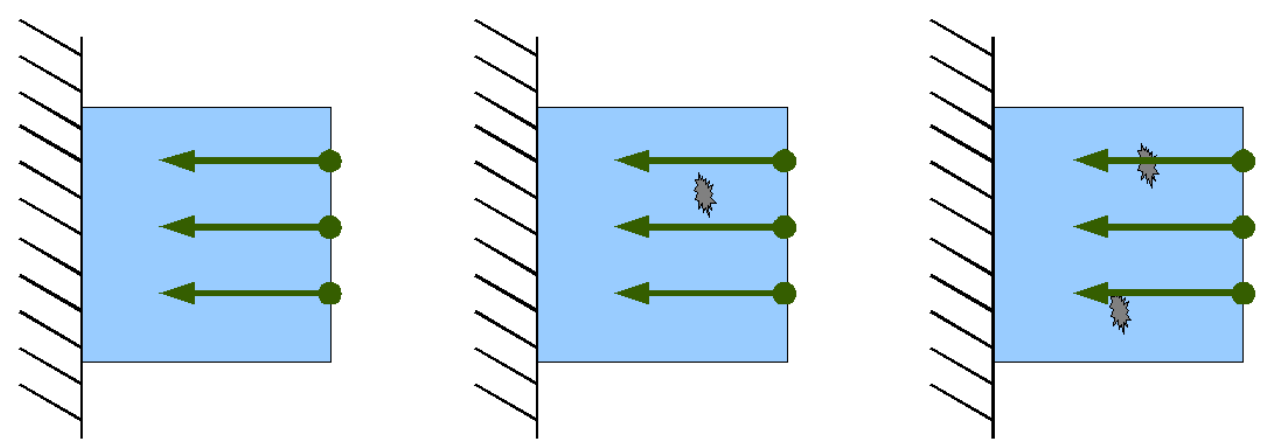

FiguRE 6. From left to right, the experiment without inclusion, two experiments with inclusions.

with

$$
\begin{aligned}
\phi(u) & =\frac{1}{2}\left(D u+D u^{T}\right), \\
\sigma(u) & =h H_{0} \phi(u),
\end{aligned}
$$

where $\sigma(u)$ is a stress distribution, $H_{0}$ is the Hooke tensor and $h(x)=1$ and $\varepsilon$ represents the material stiffness. Unlike the previous illustration with the topology optimization, this time $\varepsilon$ represents a stiff material property 

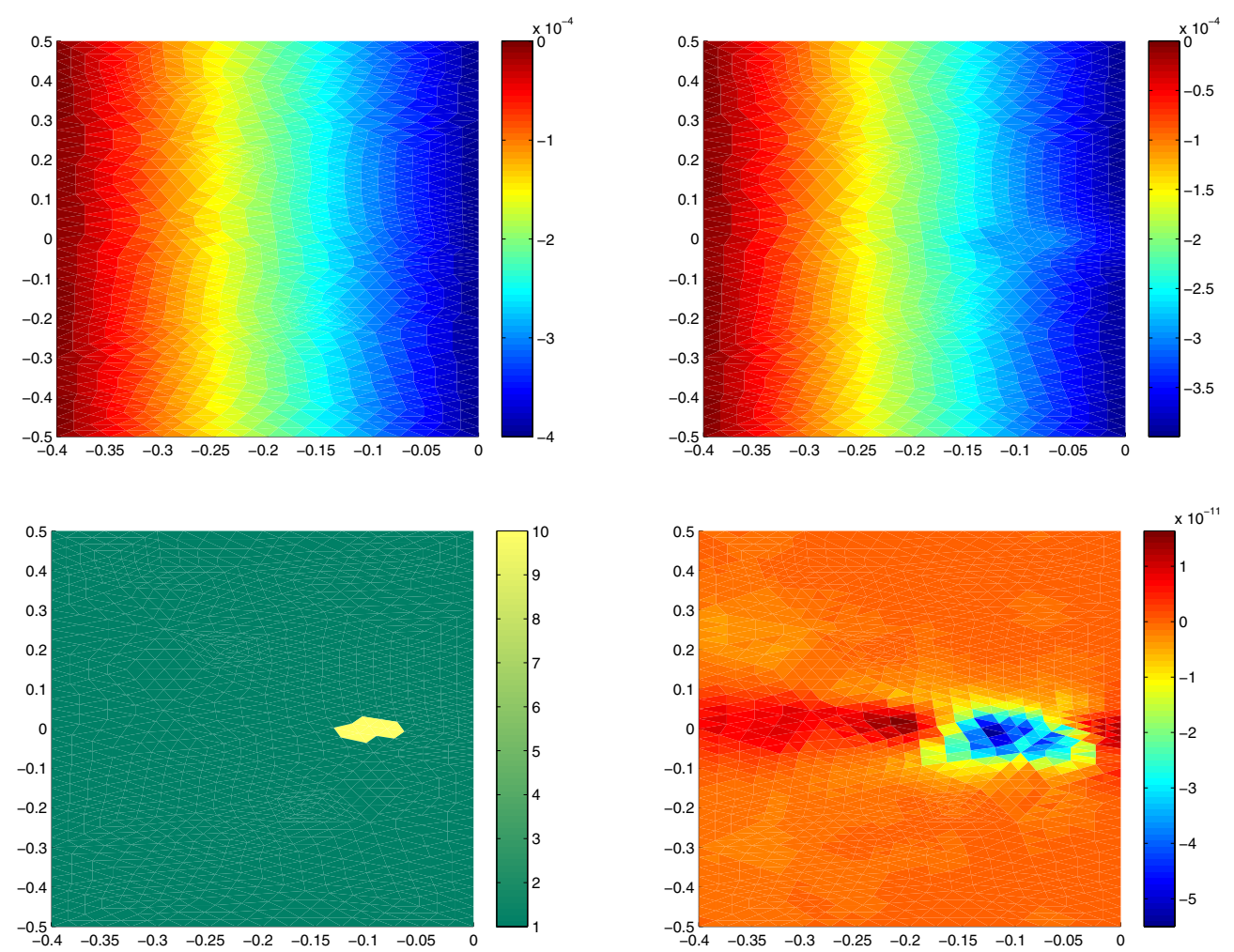

Figure 7. From left to right, in the first row, the horizontal displacements for the uniform material and the perturbed material. In the second row, the inclusion and the detection obtained with the numerical vault.

compared to the main part of the model, see Figure 6. Stiff material inhomogeneities are more difficult to detect than soft ones.

The optimization problem is to minimize the following cost function

$$
J(h)=\int_{\Omega}\left|u_{h}-u_{\mathrm{obs}}\right|^{2} \mathrm{~d} x .
$$

\subsection{Numerical results}

Two experiments are presented in this section to establish that an algorithm using the numerical vault to compute an approximation of the variations of the cost function is able to recover the localization of some material inclusions. The stiffness of the inclusion is supposed to be known.

In the first experiment, there is only one inclusion. The algorithm computes the estimation of the cost function obtained by the numerical vault for each element of the mesh. The considered element is supposed to be the inhomogeneity, the computations are only local. The domain $D$ is made of four layers of elements around the considered element. Figure 7 presents the horizontal displacements without and with the inclusion, the inclusion and the location result. The detection obtained with the numerical vault allows to detect the correct location of the inclusion and gives already an idea of its shape.

In the second experiment, there are two inclusions. The algorithm is the same as previously so it is only looking for one inclusion. Figure 8 presents the horizontal displacements without and with the inclusions, the 

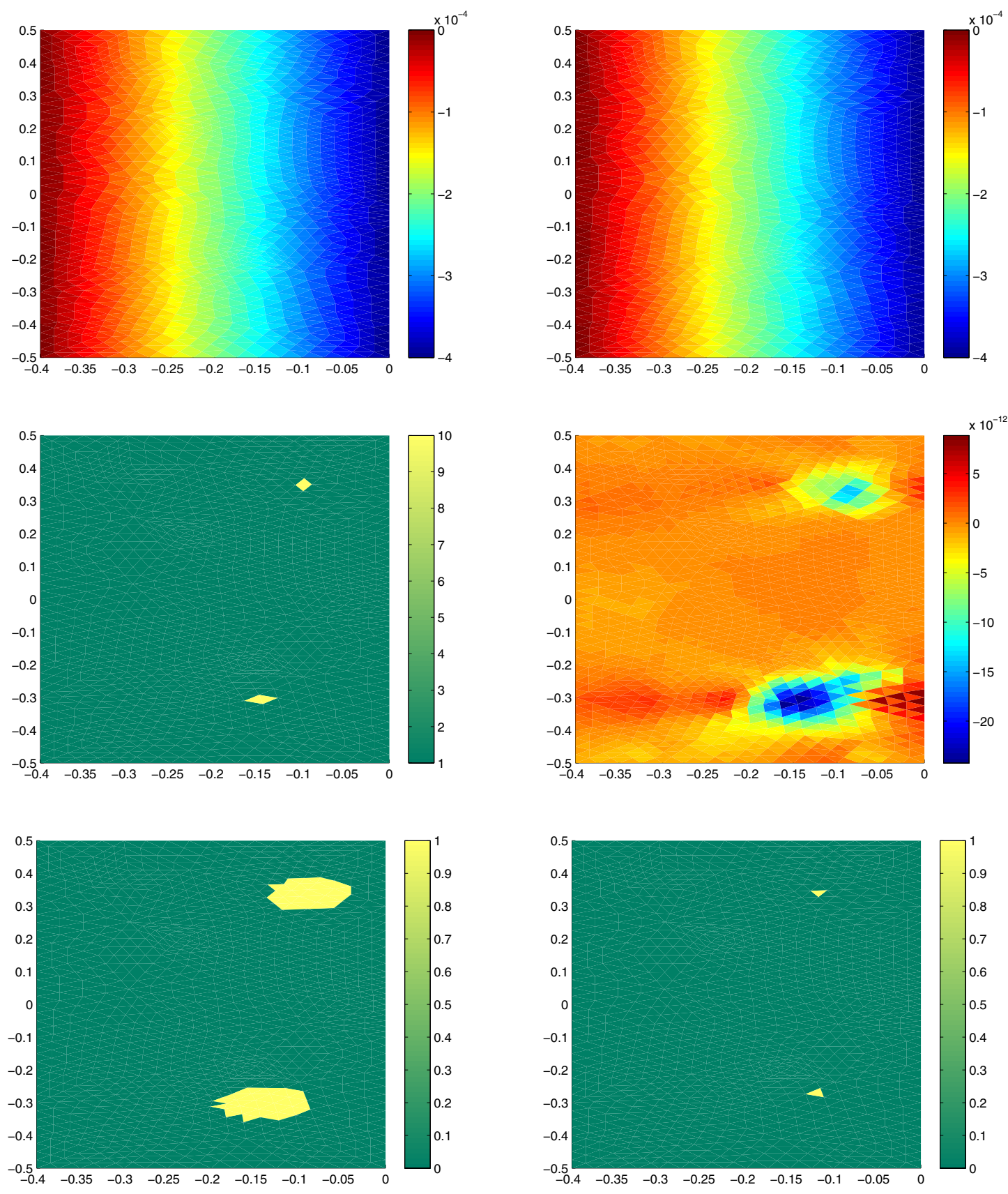

Figure 8. From left to right, in the first row, the horizontal displacements for the uniform material and the perturbed material. In the second row, the inclusions and the detection obtained with numerical vault by looking for one inclusion. In the third row, two initial detection zones and the two localization results with the numerical vault by looking for two inclusions. 
inclusions considered and the location result. The detection obtained with the numerical vault by looking for one inclusion is not fully accurate but allows us to localize two inclusions. Another set of computations is performed by creating a perturbation in each localization zone. The small size of localization zones reduces the complexity of our computations. The result is neatly improved and gives better localizations.

In both cases, the detection can be improved by using the numerical vault to test the elements surrounding the localization. It may permit to obtain a better localization and gives an idea of the inclusion shape. A mesh refinement or mesh displacement, followed by some computations with the numerical vault will also generate a better detection.

\section{IMAGE RESTORATION}

\subsection{Problem statement}

The aim of this section is to illustrate Section 4 by reconstructing a perturbed solution using the numerical vault. A classical way to restore an image $u$ from its noisy version $v$ defined in a domain $\Omega \subset \mathbb{R}^{2}$ is to solve the following PDE problem

$$
\begin{cases}-\nabla \cdot(c \nabla u)+u=v & \text { in } \Omega \\ \partial_{n} u=0 & \text { on } \partial \Omega\end{cases}
$$

where $c$ is a positive constant or a tensor [12] and $\partial_{n}$ denotes the normal derivative to $\partial \Omega$.

Topological gradient has been applied to the restoration problem in order to determine a diffusion coefficient $c$ preserving the edges $[14,16]$. In this section, the estimation obtained by the numerical vault is employed.

At a given point $x_{0} \in \Omega$, we insert a small insulating crack $\sigma_{\varepsilon}=x_{0}+\varepsilon \sigma(\mathbf{n})$ where $\sigma(\mathbf{n})$ is a straight crack and $\mathbf{n}$ is a unit vector normal to the crack. We now consider $\Omega_{\sigma}=\Omega \backslash \sigma_{\varepsilon}$ the perturbed domain created by inserting this crack. The perturbed solution $u^{\varepsilon} \in \mathrm{H}^{1}\left(\Omega_{\sigma}\right)$ satisfies

$$
\begin{cases}-\nabla \cdot\left(c \nabla u^{\varepsilon}\right)+u^{\varepsilon}=v & \text { in } \Omega, \\ \partial_{n} u^{\varepsilon}=0 & \text { on } \partial \Omega, \\ \partial_{n} u^{\varepsilon}=0 & \text { on } \sigma_{\varepsilon} .\end{cases}
$$

The corresponding variational formulation is given by

$$
\left\{\begin{array}{l}
\text { Find } u^{\varepsilon} \in \mathrm{H}^{1}\left(\Omega_{\sigma}\right) \text { such that } \\
a^{\varepsilon}\left(u^{\varepsilon}, w\right)=\ell^{\varepsilon}(w) \quad \forall w \in \mathrm{H}^{1}\left(\Omega_{\sigma}\right),
\end{array}\right.
$$

where $a^{\varepsilon}$ is the bilinear form, defined on $\mathrm{H}^{1}\left(\Omega_{\sigma}\right) \times \mathrm{H}^{1}\left(\Omega_{\sigma}\right)$ by

$$
a^{\varepsilon}(u, w)=\int_{\Omega_{\sigma}} c \nabla u \nabla w+u w \mathrm{~d} x
$$

and $\ell^{\varepsilon}$ is the linear form, defined on $\mathrm{L}^{2}\left(\Omega_{\sigma}\right)$ by

$$
\ell^{\varepsilon}(w)=\int_{\Omega_{\sigma}} v w \mathrm{~d} x .
$$




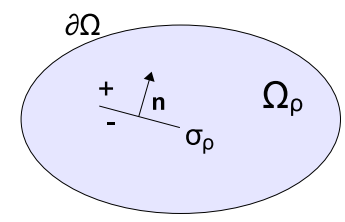

Figure 9. Cracked domain.

The domain $\Omega$ is decomposed in two parts. A part $D$ containing the perturbation and its complementary $\Omega_{0}=\Omega \backslash \bar{D}$. So the forms $a^{\varepsilon}$ and $l^{\varepsilon}$ are decomposed in the following way:

$$
\begin{gathered}
a^{\varepsilon}(u, w)=a_{\Omega_{0}}(u, w)+a_{D}^{\varepsilon}(u, w)=\int_{\Omega_{0}} c \nabla u \nabla w+u w \mathrm{~d} x+\int_{D} c \nabla u \nabla w+u w \mathrm{~d} x, \\
\ell^{\varepsilon}(w)=\ell_{\Omega_{0}}(w)+\ell_{D}^{\varepsilon}(w)=\int_{\Omega_{0}} v w \mathrm{~d} x+\int_{D} v w \mathrm{~d} x,
\end{gathered}
$$

where $a_{\Omega_{0}}$ and $l_{\Omega_{0}}$ are independent of $\varepsilon$.

The local perturbed solution $u_{u^{0}}^{\varepsilon} \in \mathrm{H}^{1}(D)$ satisfies

$$
\begin{cases}-\nabla \cdot\left(c \nabla u_{u^{0}}^{\varepsilon}\right)+u_{u^{0}}^{\varepsilon}=v & \text { in } D, \\ \partial_{n} u_{u^{0}}^{\varepsilon}=0 & \text { on } \sigma_{\varepsilon}, \\ u_{u^{0}}^{\varepsilon}=u^{0} & \text { on } \partial D .\end{cases}
$$

Let $\tilde{u}^{\varepsilon}$ be the update of $u^{0}$

$$
\tilde{u}^{\varepsilon}= \begin{cases}u_{u^{0}}^{\varepsilon} & \text { in } D \\ u^{0} & \text { in } \Omega_{0}\end{cases}
$$

The function $v^{\varepsilon}$ satisfies

$$
\begin{cases}-\nabla \cdot\left(c \nabla \tilde{u}^{\varepsilon}\right)+\tilde{u}^{\varepsilon}=v^{\varepsilon} & \text { in } \Omega_{\varepsilon}, \\ \partial_{n} \tilde{u}^{\varepsilon}=0 & \text { on } \sigma_{\varepsilon}, \\ \partial_{n} \tilde{u}^{\varepsilon}=0 & \text { on } \partial \Omega_{\varepsilon} .\end{cases}
$$

The function $\delta u^{\varepsilon}$ satisfies

$$
\begin{cases}-\nabla \cdot\left(c \nabla \delta u^{\varepsilon}\right)+\delta u^{\varepsilon}=v-v^{\varepsilon} & \text { in } \Omega, \\ \partial_{n} \delta u^{\varepsilon}=0 & \text { on } \partial \Omega .\end{cases}
$$

The approximation of $u^{\varepsilon}$ is $\tilde{\tilde{u}}^{\varepsilon}=\tilde{u}^{\varepsilon}+\delta u^{\varepsilon}$.

\subsection{Topological gradient, solving the perturbed problem}

The function $u^{0} \in \mathrm{H}^{1}(\Omega)$ is the solution of the following direct problem:

$$
\begin{cases}-\nabla \cdot\left(c \nabla u^{0}\right)+u^{0}=v & \text { in } \Omega, \\ \partial_{n} u^{0}=0 & \text { on } \partial \Omega,\end{cases}
$$

where $\partial_{n}$ denotes the normal derivative to $\partial \Omega$.

The edge detection method consists in looking for a crack $\sigma$ such that the energy $j(\varepsilon)=J_{\varepsilon}\left(u^{\varepsilon}\right)=\frac{1}{2} \int_{\Omega_{\varepsilon}}\left|\nabla u^{\varepsilon}\right|^{2}$ is as small as possible [16]. The function $u^{\varepsilon}$ is the solution of equation (7.2). This amounts to state that the energy outside the edges is as small as possible.

The cost function $j$ has the following asymptotic expansion

$$
j(\varepsilon)-j(0)=\varepsilon^{2} g\left(\mathbf{x}_{0}, \mathbf{n}\right)+o\left(\varepsilon^{2}\right),
$$


where the topological gradient $g$ is given by

$$
g\left(\mathbf{x}_{0}, \mathbf{n}\right)=-\pi c\left(\nabla u^{0}\left(\mathbf{x}_{0}\right) \cdot \mathbf{n}\right)\left(\nabla p^{0}\left(\mathbf{x}_{0}\right) \cdot \mathbf{n}\right)-\pi\left|\nabla u^{0}\left(\mathbf{x}_{0}\right) \cdot \mathbf{n}\right|^{2} .
$$

The function $p^{0}$ is the solution to the adjoint problem:

$$
\begin{cases}-\nabla \cdot\left(c \nabla p^{0}\right)+p^{0}=-\partial_{u} J_{0}\left(u^{0}\right) & \text { in } \Omega, \\ \partial_{n} p^{0}=0 & \text { on } \partial \Omega .\end{cases}
$$

The functions $u^{0}$ and $p^{0}$ are calculated in the initial domain without cracks. The topological gradient can be written $g(\mathbf{x}, \mathbf{n})=\mathbf{n}^{T} M(\mathbf{x}) \mathbf{n}$, where $M(\mathbf{x})$ is the $2 \times 2$ symmetric matrix defined by

$$
M(\mathbf{x})=-\pi c \frac{\nabla u^{0}(\mathbf{x}) \nabla p^{0}(\mathbf{x})^{T}+\nabla p^{0}(\mathbf{x}) \nabla u^{0}(\mathbf{x})^{T}}{2}-\pi \nabla u^{0}(\mathbf{x}) \nabla u^{0}(\mathbf{x})^{T} .
$$

For a given $\mathbf{x}, g(\mathbf{x}, \mathbf{n})$ takes its minimal value when $\mathbf{n}$ is the eigenvector associated to the lowest eigenvalue $\lambda_{\min }(\mathbf{x})$ of $M(\mathbf{x})$. This value is the topological gradient associated to the optimal orientation of the crack $\sigma_{\varepsilon}$ at the location $\mathbf{x}$.

The edges are located at points $\mathbf{x}$ where $\lambda_{\min }(\mathbf{x})$ is the most negative and their orientation is given by the corresponding eigenvector.

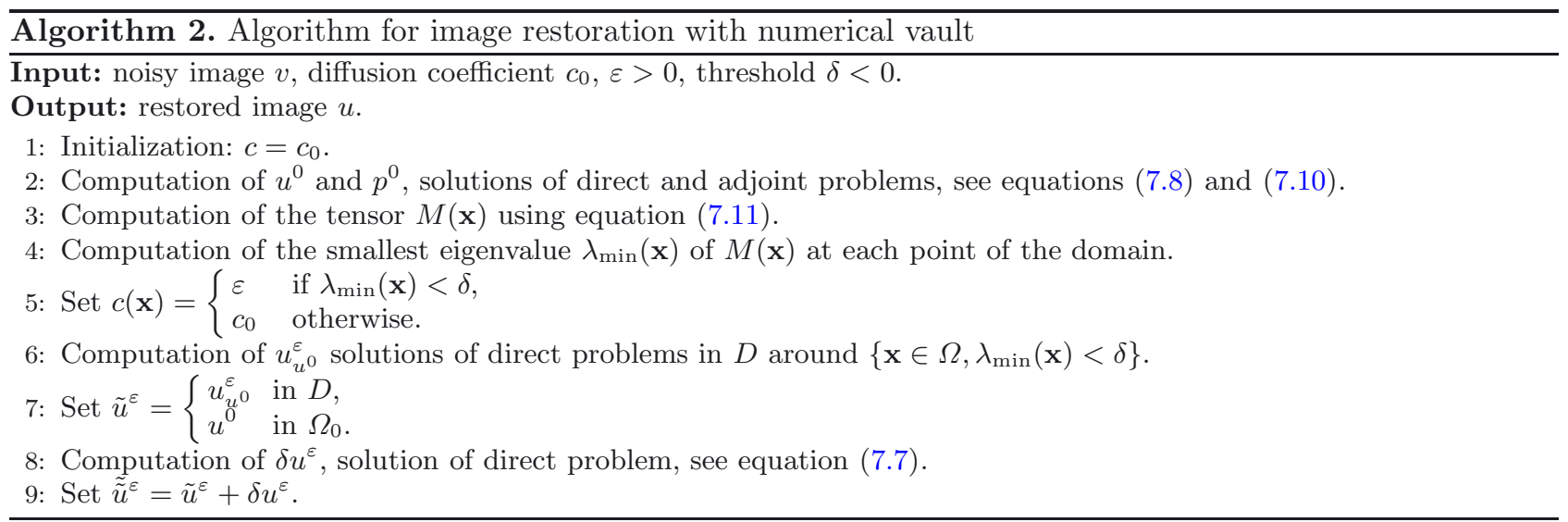

\subsection{Numerical results in image restoration}

The Peak-Signal-to-Noise Ratio (PSNR) and the Structural SIMilarity (SSIM) [37] are used to quantify the restorations. The PSNR formula is the following

$$
P S N R=10 \log _{10}\left(\frac{255^{2} m n}{\sum_{i=0}^{m-1} \sum_{j=0}^{n-1}[I(i, j)-K(i, j)]^{2}}\right),
$$

where $I$ and $K$ are the images of dimension $m \times n$ which are compared. The SSIM follows this equation:

$$
S S I M=\frac{\left(2 \mu_{I} \mu_{K}+c_{1}\right)\left(2 \sigma_{I, K}+c_{2}\right)}{\left(\mu_{I}^{2}+\mu_{K}^{2}+c_{1}\right)\left(\sigma_{I}^{2}+\sigma_{K}^{2}+c_{2}\right)},
$$

where $\mu_{I}$ is the average of $I, \mu_{K}$ is the average of $K, \sigma_{I}^{2}$ is the variance of $I, \sigma_{K}^{2}$ is the variance of $K, \sigma_{I, K}^{2}$ is the covariance of $I$ and $K, c_{1}=\left(k_{1} L\right)^{2}$ and $c_{2}=\left(k_{2} L\right)^{2}$ two variables to stabilize the division by a weak

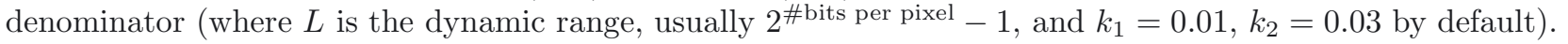



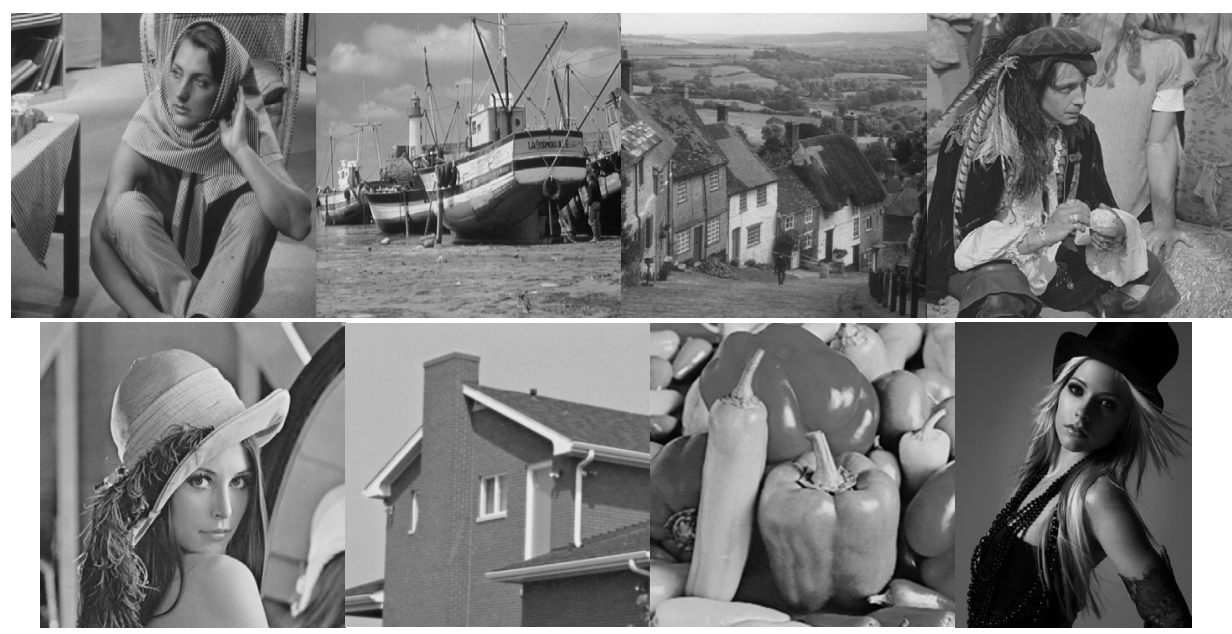

Figure 10. Set of gray-level images used for tests.

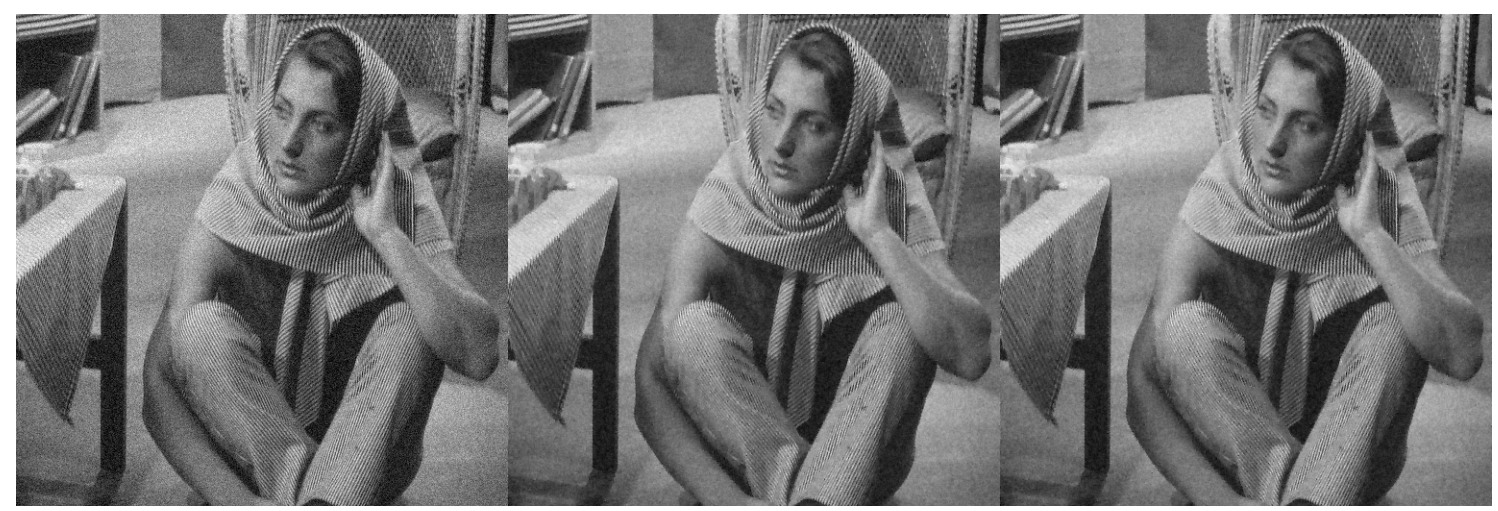

FiguRE 11. From left to right, the Barbara noisy image with $\sigma=15$, the reconstructions with respectively the isotropic diffusion and the numerical vault.

Figure 10 presents the set of gray-level images: Barbara, Boat, Hill, Man and Lena $(512 \times 512)$; House and Peppers $(256 \times 256)$; Girl $(462 \times 357)$ used for the tests. The domain $D$ is made of one layer of elements around the edge.

Table 3 presents the Barbara restoration results from images perturbed by additive Gaussian noises of standard deviation $\sigma=5,15$ and 25. The first approximation (7.5) (FA) is not sufficient. The second approximation (SA) has similar results. Figure 11 shows the noisy image and illustrates the similarity between the restorations.

Table 4 presents the other image restoration results from images perturbed by additive Gaussian noises of standard deviation $\sigma=5,15$ and 25. The restorations giving by the direct equation and by the numerical vault are nearly equivalent. Figure 12 gives another visual results, the noisy image and illustrates the similarity between the two restorations for the Girl image.

The numerical vault approach is faster than the classical method. For the reconstruction with the isotropic diffusion (ID), equation (7.1) needs to be solved with a diffusion coefficient $c$ that depends on the topological gradient. This resolution is made with a preconditioned conjugate gradient (PCG) method preconditioned by a DCT solver and a constant diffusion coefficient $c$ (see [15] for details). The method with the numerical vault uses 


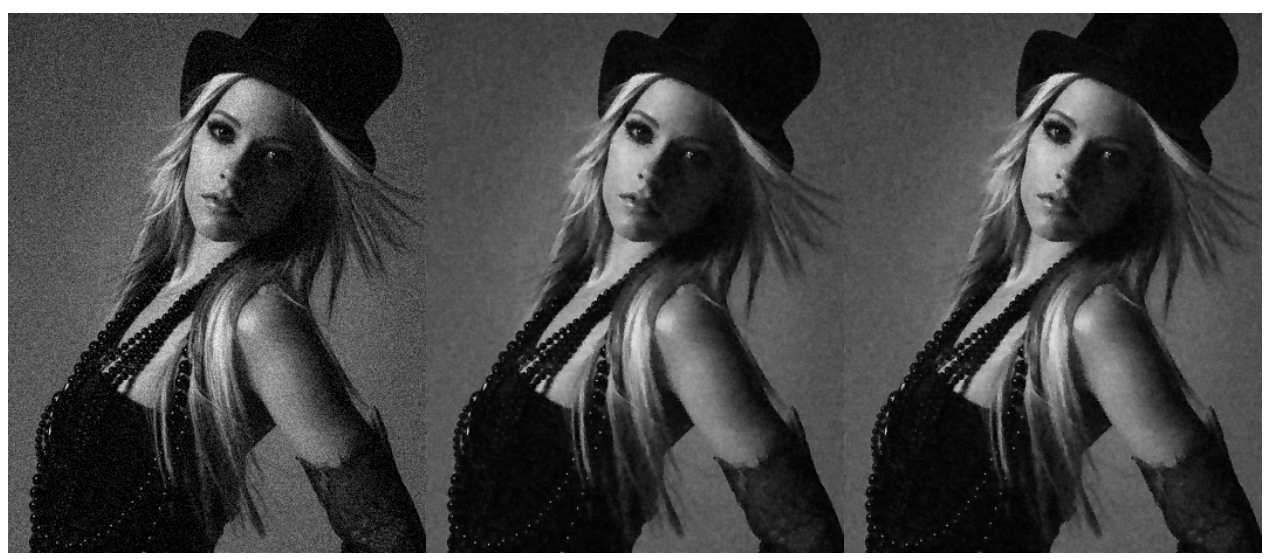

Figure 12. From left to right, the Girl noisy image with $\sigma=15$, the reconstructions obtained with respectively the isotropic diffusion and the numerical vault.

TABLE 3. Comparative results of the restoration using the isotropic diffusion (ID), the first approximation (7.5) (FA) and the second approximation (SA) obtained with Algorithm 2.

\begin{tabular}{|c|c|c|c|c|c|c|}
\hline Barbara & \multicolumn{2}{|c|}{$\sigma=5$} & \multicolumn{2}{c|}{$\sigma=15$} & \multicolumn{2}{c|}{$\sigma=25$} \\
$512 \times 512$ & PSNR & SSIM & PSNR & SSIM & PSNR & SSIM \\
\hline Noisy & 34.13 & 0.881 & 24.62 & 0.560 & 20.25 & 0.386 \\
\hline ID & 35.78 & 0.942 & 27.92 & 0.760 & 25.32 & 0.638 \\
\hline FA & 34.87 & 0.933 & 27.20 & 0.747 & 24.93 & 0.630 \\
\hline SA & 35.74 & 0.938 & 27.87 & 0.758 & 25.28 & 0.638 \\
\hline
\end{tabular}

TABLE 4. Comparative results of the restoration using the isotropic diffusion (ID) and the second approximation (SA) obtained with Algorithm 2.

\begin{tabular}{|c|c|c|c|c|c|c|c|}
\cline { 3 - 8 } \multicolumn{2}{c|}{} & \multicolumn{2}{c|}{$\sigma=5$} & \multicolumn{2}{c|}{$\sigma=15$} & \multicolumn{2}{c|}{$\sigma=25$} \\
\cline { 3 - 8 } \multicolumn{2}{c|}{} & PSNR & SSIM & PSNR & SSIM & PSNR & SSIM \\
\hline \multirow{3}{*}{ Boat } & Noisy & 34.18 & 0.885 & 24.63 & 0.537 & 20.29 & 0.348 \\
\cline { 2 - 8 } & ID & 36.14 & 0.926 & 29.97 & 0.797 & 27.56 & 0.709 \\
\cline { 2 - 8 } & SA & 36.14 & 0.926 & 29.80 & 0.795 & 27.40 & 0.705 \\
\hline \multirow{3}{*}{ Lill } & Noisy & 34.13 & 0.888 & 24.63 & 0.536 & 20.27 & 0.328 \\
\cline { 2 - 8 } & ID & 36.17 & 0.930 & 30.32 & 0.794 & 28.25 & 0.711 \\
\cline { 2 - 8 } & SA & 36.17 & 0.930 & 30.22 & 0.792 & 28.16 & 0.709 \\
\hline \multirow{3}{*}{ Man } & Noisy & 34.13 & 0.844 & 24.62 & 0.451 & 20.21 & 0.272 \\
\cline { 2 - 8 } & ID & 37.24 & 0.931 & 31.42 & 0.819 & 29.14 & 0.754 \\
\cline { 2 - 8 } & SA & 37.21 & 0.931 & 31.32 & 0.818 & 29.03 & 0.752 \\
\hline \multirow{3}{*}{ House } & Noisy & 34.13 & 0.878 & 24.60 & 0.522 & 20.25 & 0.332 \\
\cline { 2 - 8 } & ID & 36.44 & 0.937 & 30.27 & 0.807 & 28.07 & 0.729 \\
\cline { 2 - 8 } & SA & 36.43 & 0.937 & 30.15 & 0.805 & 27.91 & 0.726 \\
\cline { 2 - 8 } & Noisy & 34.20 & 0.839 & 24.64 & 0.447 & 20.19 & 0.278 \\
\cline { 2 - 8 } & ID & 37.34 & 0.926 & 31.59 & 0.823 & 28.95 & 0.753 \\
\hline \multirow{3}{*}{ Geppers } & 37.24 & 0.925 & 31.24 & 0.820 & 28.52 & 0.747 \\
\cline { 2 - 8 } & Noisy & 34.14 & 0.870 & 24.60 & 0.523 & 20.35 & 0.352 \\
\cline { 2 - 8 } & ID & 37.48 & 0.954 & 30.93 & 0.859 & 28.10 & 0.786 \\
\cline { 2 - 8 } & SA & 37.38 & 0.954 & 30.69 & 0.857 & 27.90 & 0.782 \\
\cline { 2 - 8 } & Noisy & 34.17 & 0.815 & 25.12 & 0.437 & 20.81 & 0.271 \\
\cline { 2 - 7 } & ID & 37.72 & 0.944 & 31.04 & 0.786 & 28.22 & 0.698 \\
\hline & SA & 37.59 & 0.943 & 30.91 & 0.785 & 28.07 & 0.695 \\
\hline
\end{tabular}


TABLE 5. Comparative results of number of iteration steps of PCG using the isotropic diffusion (ID) and the second approximation (SA) obtained with Algorithm 2.

\begin{tabular}{|c|c|c|c|c|c|c|}
\cline { 2 - 7 } \multicolumn{1}{c|}{ PCG iter. } & \multicolumn{2}{c|}{$\sigma=5$} & \multicolumn{2}{c|}{$\sigma=15$} & \multicolumn{2}{c|}{$\sigma=25$} \\
\hline Image & ID & SA & ID & SA & ID & SA \\
\hline Barbara & 10 & 8 & 10 & 7 & 9 & 5 \\
\hline Man & 9 & 8 & 9 & 5 & 10 & 4 \\
\hline
\end{tabular}

the same tools to compute the solution of equation (7.7) with a constant diffusion coefficient $c$. The computations to obtain the first update are only local, see equation (7.4), and are negligible compared to the problem size.

Table 5 presents the number of iterations of PCG using the isotropic diffusion (ID) and the second approximation obtained with the algorithm (SA) from images perturbed by additive Gaussian noises of standard deviation $\sigma=5,15$ and 25. For the same tolerance, in term of PCG iterations, the resolution with the numerical vault is faster than the classical method.

\section{Conclusion}

In this work, the adjoint method has been extended with a new method named the numerical vault. The theoretical sections justify that the numerical vault is able to capture the variation of the cost function in a better way than the previous adjoint method. They also demonstrate that the vault method can be applied to update the solution of a singularly perturbed problem.

The numerical applications on mesh perturbation and topological and continuous variations show the superiority of the results obtained using the vault method compared to those obtained using the adjoint method. The curves presented in the numerical applications have illustrated that the numerical vault captures higher order of the variation compared to the adjoint method. As a result, inclusions in elastography could be successfully localized. To solve these problems, only a very simple direct solution and some local computations have been performed. Major advantages of the method are that the numerical vault is non invasive and can be used with a parallel computing implementation.

The second interest is to offer a new method to resolve direct equations, avoiding the resolution of the full direct perturbed equation, which can be costly in computing time, when only local zones are affected by modifications of the equation operator. In the image applications, the purpose was not to obtain good restoration results - they can be improved by using an anisotropic diffusion scheme [28] - but to demonstrate that the approximation created with the numerical vault is equivalent to the direct solution.

The numerical vault is a universal tool that can be applied to many different linear problems as long as the hypothesis holds. Research in nonlinear applications is under consideration.

\section{REFERENCES}

[1] G. Allaire, F. de Gournay, F. Jouve and A.-M. Toader, Structural optimization using topological and shape sensitivity via a level set method. Control Cybern. 34 (2005) 59-80.

[2] H. Ammari and H. Kang, High-order terms in the asymptotic expansions of the steady-state voltage potentials in the presence of conductivity inhomogeneities of small diameter. SIAM J. Math. Anal. 34 (2003) 1152-1166.

[3] H. Ammari and H. Kang, Reconstruction of small inhomogeneities from boundary measurements. Lect. Notes Math. 1846 (2004).

[4] H. Ammari and J.K. Seo, An accurate formula for the reconstruction of conductivity inhomogeneities. Adv. Appl. Math. 30 (2003) 679-705.

[5] H. Ammari, S. Moskow and M.S. Vogelius, Boundary integral formulae for the reconstruction of electric and electromagnetic inhomogeneities of small volume. ESAIM: COCV 9 (2003) 49-66.

[6] H. Ammari, E. Iakovleva, D. Lesselier and G. Perrusson, MUSIC-type electromagnetic imaging of a collection of small threedimensional inclusions. SIAM J. Sci. Comput. 29 (2007) 674-709.

[7] H. Ammari, E. Bonnetier, Y. Capdeboscq, M. Tanter and M. Fink, Electrical impedance tomography by elastic deformation. SIAM J. Appl. Math. 68 (2008) 1557-1573. 
[8] H. Ammari, P. Garapon, H. Kang and H. Lee, A method of biological tissues elasticity reconstruction using magnetic resonance elastography measurements. Quart. Appl. Math. 66 (2008) 139-175.

[9] H. Ammari, P. Garapon, H. Kang and H. Lee, Separation of scales in elasticity imaging: a numerical study. J. Comput. Math. 28 (2010) 354-370.

[10] S. Amstutz, M. Masmoudi and B. Samet, The topological asymptotic for the Helmoltz equation. SIAM J. Control Optim. 42 (2003) 1523-1544.

[11] S. Amstutz, I. Horchani and M. Masmoudi, Crack detection by the topological gradient method. Control Cybern. 34 (2005) 81-101.

[12] G. Aubert and P. Kornprobst, Mathematical Problems in Image Processing: Partial Differential Equations and the Calculus of Variations. Appl. Math. Sci. 147 (2001).

[13] D. Auroux and M. Masmoudi, A one-shot inpainting algorithm based on the topological asymptotic analysis. Comput. Appl. Math. 25 (2006) 251-267.

[14] D. Auroux and M. Masmoudi, Image processing by topological asymptotic expansion. J. Math. Imag. Vision 33 (2009) $122-134$.

[15] D. Auroux and M. Masmoudi, Image processing by topological asymptotic analysis. ESAIM: Proc. Math. Methods Imag. Inverse Probl. 26 (2009) 24-44.

[16] L.J. Belaid, M. Jaoua, M. Masmoudi and L. Siala, Image restoration and edge detection by topological asymptotic expansion. C. R. Acad. Sci. Paris 342 (2006) 313-318.

[17] M. Bonnet, Higher-order topological sensitivity for 2-d potential problems. application to fast identification of inclusions. Int. J. Solids Struct. 46 (2009) 2275-2292.

[18] M. Bonnet, Fast identification of cracks using higher-order topological sensitivity for 2-d potential problems. Special issue on the advances in mesh reduction methods. In honor of Professor Subrata Mukherjee on the occasion of his 65th birthday. Eng. Anal. Bound. Elem. 35 (2011) 223-235.

[19] Y. Capdeboscq and M.S. Vogelius, A general representation formula for boundary voltage perturbations caused by internal conductivity inhomogeneities of low volume fraction. ESAIM: M2AN 37 (2003) 159-173.

[20] Y. Capdeboscq and M.S. Vogelius, Optimal asymptotic estimates for the volume of internal inhomogeneities in terms of multiple boundary measurements. ESAIM: M2AN 37 (2003) 227-240.

[21] J. Fehrenbach and M. Masmoudi, Coupling topological gradient and Gauss-Newton methods, in IUTAM Symposium on Topological Design Optimization. Edited by M.P. Bendsoe, N. Olhoff and O. Sigmund. Springer (2006).

[22] J. Fehrenbach, M. Masmoudi, R. Souchon and P. Trompette, Detection of small inclusions using elastography. Inverse Probl. 22 (2006) 1055-1069.

[23] S. Garreau, P. Guillaume and M. Masmoudi, The topological asymptotic for pde systems: the elasticity case. SIAM J. Control Optim. 39 (2001) 1756-1778.

[24] P. Guillaume and M. Hassine, Removing holes in topological shape optimization. ESAIM: COCV 14 (2008) $160-191$.

[25] P. Guillaume and K. Sid Idris, The topological asymptotic expansion for the Dirichlet problem. SIAM J. Control Optim. 41 (2002) 1042-1072.

[26] P. Guillaume and K. Sid Idris, The topological sensitivity and shape optimization for the Stokes equations. SIAM J. Control Optim. 43 (2004) 1-31.

[27] M. Hassine, S. Jan and M. Masmoudi, From differential calculus to 0-1 topological optimization. SIAM, J. Control Optim. 45 (2007) 1965-1987.

[28] S. Larnier and J. Fehrenbach, Edge detection and image restoration with anisotropic topological gradient, in IEEE International Conference on Acoustics Speech and Signal Processing (ICASSP) (2010) 1362-1365.

[29] L. Martin, Conception aérodynamique robuste. Ph.D. thesis, Université Paul Sabatier, Toulouse, France (2011).

[30] M. Masmoudi, The topological asymptotic, in Computational Methods for Control Applications, GAKUTO International Series, edited by R. Glowinski, H. Karawada and J. Periaux. Math. Sci. Appl. 16 (2001) 53-72.

[31] B. Mohammadi and O. Pironneau, Shape optimization in fluid mechanics. Annu. Rev. Fluid Mech. 36 (2004) $255-279$.

[32] J. Ophir, I. Céspedes, H. Ponnekanti, Y. Yazdi and X. Li, Elastography: a quantitative method for imaging the elasticity of biological tissues. Ultrason. Imag. 13 (1991) 111-134.

[33] J. Ophir, S. Alam, B. Garra, F. Kallel, E. Konofagou, T. Krouskop, C. Merritt, R. Righetti, R. Souchon, S. Srinivan and T. Varghese, Elastography: imaging the elastic properties of soft tissues with ultrasound. J. Med. Ultrason. 29 (2002) $155-171$.

[34] B. Samet, The topological asymptotic with respect to a singular boundary perturbation. C. R. Math. 336 (2003) $1033-1038$.

[35] A. Schumacher, Topologieoptimisierung von Bauteilstrukturen unter Verwendung von Lopchpositionierungkrieterien. Ph.D. thesis, Universitat-Gesamthochschule Siegen, Germany (1995).

[36] J. Sokolowski and A. Zochowski, On the topological derivative in shape optimization. SIAM J. Control Optim. 37 (1999) 1251-1272.

[37] Z. Wang, A.C. Bovik, H.R. Sheikh and E.P. Simoncelli, Image quality assessment: from error visibility to structural similarity. IEEE Trans. Image Process. 13 (2004) 600-612. 